\title{
Larval ecology of mosquitoes in sylvatic arbovirus foci in southeastern Senegal
}

Diawo Diallo ${ }^{1 *}$, Cheikh T Diagne ${ }^{1}$, Kathryn A Hanley², Amadou A Sall ${ }^{3}$, Michaela Buenemann ${ }^{4}$, Yamar Ba ${ }^{1}$, Ibrahima Dia ${ }^{1}$, Scott C Weaver ${ }^{5}$ and Mawlouth Diallo ${ }^{1}$

\begin{abstract}
Background: Although adult mosquito vectors of sylvatic arbovirus [yellow fever (YFV), dengue-2 (DENV-2) and chikungunya (CHIKV)] have been studied for the past 40 years in southeastern Senegal, data are still lacking on the ecology of larval mosquitoes in this area. In this study, we investigated the larval habitats of mosquitoes and characterized their seasonal and spatial dynamics in arbovirus foci.

Methods: We searched for wet microhabitats, classified in 9 categories, in five land cover classes (agriculture, forest, savannah, barren and village) from June, 2010 to January, 2011. Mosquito immatures were sampled monthly in up to 30 microhabitats of each category per land cover and bred until adult stage for determination.

Results: No wet microhabitats were found in the agricultural sites; in the remaining land covers immature stages of 35 mosquito species in 7 genera were sampled from 9 microhabitats (tree holes, fresh fruit husks, decaying fruit husks, puddles, bamboo holes, discarded containers, tires, rock holes and storage containers). The most abundant species was Aedes aegypti formosus, representing 30.2\% of the collections, followed by 12 species, representing each more than $1 \%$ of the total, among them the arbovirus vectors Ae. vittatus (7.9\%), Ae. luteocephalus (5.7\%), Ae. taylori (5.0\%), and Ae. furcifer (1.3\%). Aedes aegypti, Cx. nebulosus, Cx. perfuscus, Cx. tritaeniorhynchus, Er. chrysogster and Ae. vittatus were the only common species collected from all land covers. Aedes furcifer and Ae. taylori were collected in fresh fruit husks and tree holes. Species richness and dominance varied significantly in land covers and microhabitats. Positive associations were found mainly between Ae. furcifer, Ae. taylori and Ae. luteocephalus. A high proportion of potential enzootic vectors that are not anthropophilic were found in the larval mosquito fauna.
\end{abstract}

Conclusions: In southeastern Senegal, Ae. furcifer and Ae. taylori larvae showed a more limited distribution among both land cover and microhabitat types than the other common species. Uniquely among vector species, Ae. aegypti formosus larvae occurred at the highest frequency in villages. Finally, a high proportion of the potential non-anthropophilic vectors were represented in the larval mosquito fauna, suggesting the existence of unidentified sylvatic arbovirus cycles in southeastern Senegal.

Keywords: Mosquito larvae, Sylvatic arbovirus vectors, Microhabitats, Land covers, Species association, Aedes furcifer,Aedes taylori, Aedes aegypti formosus, Southeastern Senegal

\footnotetext{
* Correspondence: diawod@yahoo.com

'Unité d'entomologie médicale, Institut Pasteur de Dakar, Dakar, Sénégal

Full list of author information is available at the end of the article
} 


\section{Background}

Southeastern Senegal (West Africa) is endemic for several arboviruses including dengue-2 and yellow fever (genus Flavivirus, family Flaviviridae) and chikungunya (genus Alphavirus, family Togaviridae) viruses that occur in sylvatic, enzootic transmission cycles between primates and arboreal mosquitoes [1-3]. The first evidence of sylvatic transmission of dengue-2 virus (DENV-2) in the area was virus isolation from a human in 1970 about $60 \mathrm{~km}$ from the Senegalese capital Dakar, and from pools of Aedes luteocephalus caught in a forest gallery near the town of Kédougou in southeastern Senegal [4]. After that, 5 amplifications of the sylvatic cycle were detected between 1980 and 2000 in Kédougou. During these amplifications, large numbers of DENV-2 strains were isolated from mosquitoes, mainly from $A e$. furcifer, Ae. luteocephalus, Ae. taylori, Ae. aegypti formosus, and Ae. vitattus, one strain from the serum of a wild patas monkey (Erythrocebus patas), and four strains from human sera [5-8]. The sylvatic cycle of chikungunya virus (CHIKV) in southeastern Senegal is very similar to that of DENV-2. Indeed, although CHIKV has been isolated from 11 mosquito species and 3 different monkeys species during amplifications of the sylvatic cycle in the region, the data indicate that the main vectors (Ae. furcifer, Ae. taylori, Ae. luteocephalus) as well as vertebrate hosts (monkeys and humans) are the same as those for DENV-2. However, transmission of CHIKV may differ in subtle ways from that of sylvatic DENV-2, due to the possible existence of additional CHIKV vectors and vertebrate hosts other than monkeys, such as galagos (Galago senegalensis), palm squirrels (Xerus erythropus), and bats (Scotophillus sp) [1].

The identification of a sylvatic cycle of yellow fever virus (YFV) in southeastern Senegal $[9,10]$, led to the establishment of a surveillance program that documented the recurrence of the epizootic amplifications by the isolation of virus from mosquitoes and the detection of antibodies in simian and human sera at 4-6 year intervals, during the rainy season [11]. A three year survey, consisting of monthly 25-hour human landing collections of the mosquito fauna in a transect from a forestgallery to the nearest village, showed that only 4 species (Ae. luteocephalus, Ae vittatus, Ae. furcifer and Ae. taylori) were attracted to humans [12]. Other species were considered as non-anthropophilic. That study also showed that these species were host-seeking in the evening and that the evening collection was representative of the entire mosquito fauna.

While sylvatic transmission of these viruses is relatively well characterized in Senegal, some aspects of the ecology of their vectors are still poorly understood. Notably, only one study has been devoted to the ecology of the larval stages of arbovirus vectors [13]. Moreover, that study was limited to a single gallery forest, while there are many other land cover classes in the area. We have recently reported the distribution and abundance of adult mosquitoes potentially involved in the sylvatic cycle of CHIKV in southeastern Senegal, as well as their levels of infection in the five most abundant land cover elements (forest, savanna, agriculture, barren and village) [14]. Potential vectors are found in each of the land cover classes, but Ae. furcifer was the only species that occured in all land cover types and also entered villages to feed on humans. Thus, this species is probably the most important bridge vector between forest circulation and human populations. However, the presence of a host seeking vector in a land cover type does not always mirror the distribution of its conspecific larvae.

In addition, some non-anthropophilic mosquito species, scarce or absent in a previous 25 hour human landing collection, and probably feeding mainly on animals that have been associated with YFV, DENV-2 and CHIKV, may have large populations in the Kédougou area [2]. Understanding the larval ecology of these vectors is of particular importance for monitoring and controlling the circulation and spillover of these sylvatic viruses. An investigation of these parameters will allow us to better understand the transmission cycles and therefore the epidemiology of these viruses. Furthermore, knowledge of larval vector ecology is a key factor in risk assessment and establishment of effective control strategies and tools, because the most effective method for controlling vector populations is to control the immature stages in their aquatic habitats before they emerge as adults.

The aim of this work was therefore to identify the larval habitats of potential arbovirus vectors in the Kédougou area and characterize their seasonal and spatial dynamics.

\section{Methods}

\section{Study area}

Our study was undertaken in the Kédougou region (Figure 1) located in southeastern Senegal $\left(12^{\circ} 33 \mathrm{~N}, 12^{\circ}\right.$ $11 \mathrm{~W})$. The annual rainfall ranges from 1200 to 1300 $\mathrm{mm}$, with one rainy season between May and November, and the topography is hilly. Mean temperatures vary from $33-39.5^{\circ} \mathrm{C}$ during the year. Kédougou lies in a transition zone between the dry tropical forest and the savannah belt. A mosaic of forest, forest galleries and savannahs constitute the natural vegetation. The human population of the region is ca. 80,000 and is primarily rural (84\%) with a low overall density of inhabitants $\left(4 / \mathrm{km}^{2}\right)$, mostly living in small, dispersed villages averaging 60 inhabitants. The fauna encountered is very diverse including three monkey species, the Guinea baboon (Papio papio), the patas monkey ( $E$. patas), the African green monkey 


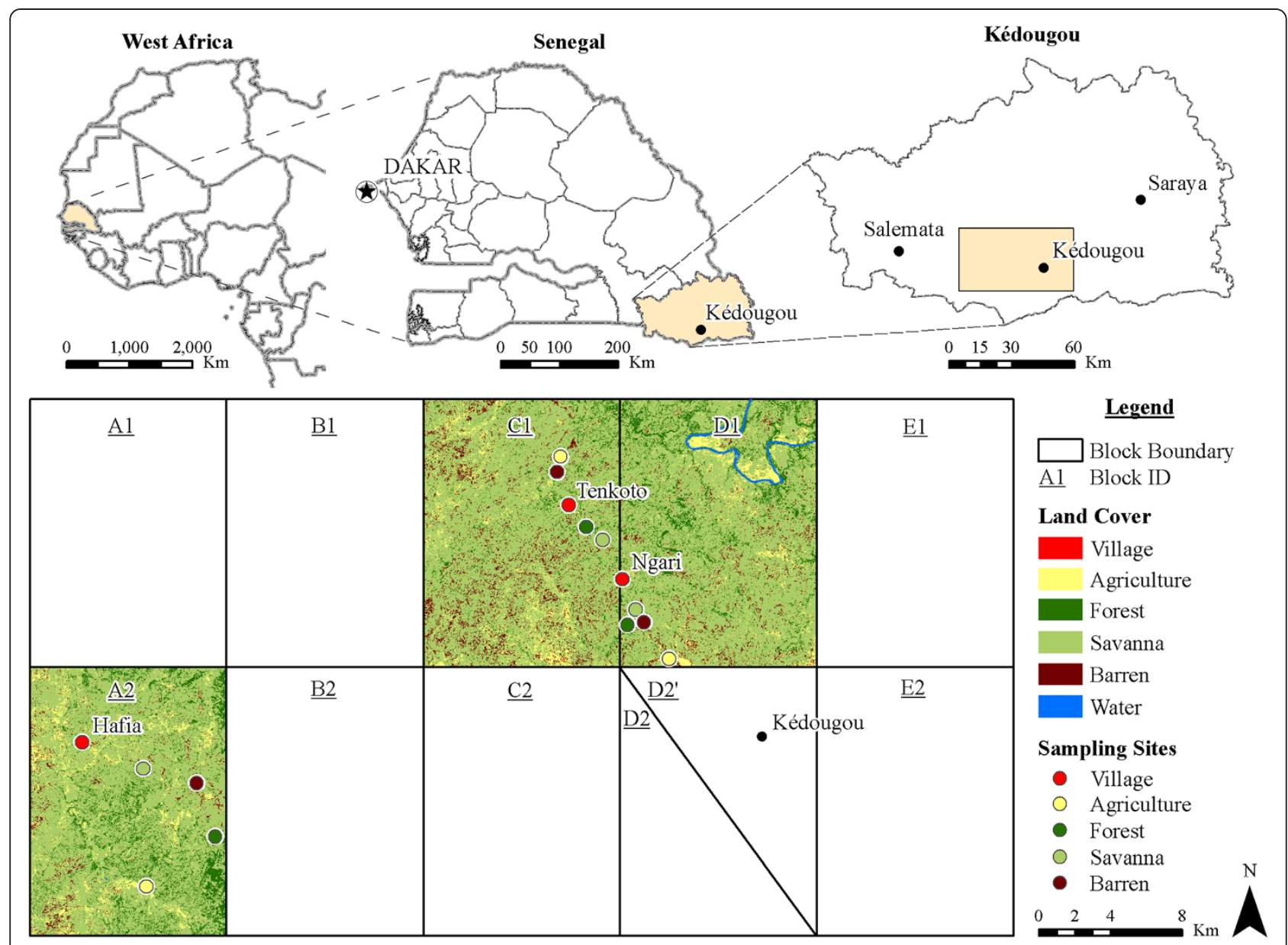

Figure 1 Location of the study site. The rectangle in the upper right map corresponds to the 1,650 $\mathrm{Km}^{2}$ divided in ten blocks (A2-E2) below. Data were collected in each of the five land covers (agriculture, barren, village, savannah and forest) in the blocks A2, C1 and D1.

(Cercopithecus sabaeus), and one ape, the chimpanzee (Pan troglodytes).

\section{Larval habitat selection and classification}

An area of $1650 \mathrm{~km}^{2}$ (30 km in N-S direction; $55 \mathrm{~km}$ in $\mathrm{E}-\mathrm{W}$ direction) of the Kédougou region (Figure 1) was divided into 10 blocks of roughly equal size. In each block, 5 different types of land cover, classified as forest, barren, savannah, agriculture and village, were defined by remote sensing and geospatial analyses, and one sampling site was chosen in each land cover class as described previously [14]. Based on previous data on the distribution and abundance of adult mosquitoes [14], blocks A2, C1 and D1 were chosen for this study of larval ecology. In a preliminary survey, all existing natural and artificial cavities or containers with the potential to hold water, in the different land cover classes, were recorded. These habitats were classified based on the origin, microhabitat, material and/or container type as decaying fruit husks, fresh fruit husks, puddles, tree holes, bamboo holes, tires, rocks-holes, discarded containers and storage containers (Figure 2). These habitat types were described as follows: 1) decaying fruit husks from the past year's production of Saba senegalensis (Apocynacées), which are thick and rigid, with a globally hemispherical shape, black colored and of small size (less than $10 \mathrm{~cm}$ in diameter); 2) fresh fruit husks from the current year production of the same plant that are less rigid, yellow colored and hold different water quality (colored and acid) compared to the decaying fruit husk; 3) tree holes were rot and pan holes of different shapes and volume located from 0 to $2 \mathrm{~m}$ above the ground level; 4) puddles were temporary small water collections that formed on the ground after rainfall and in plastic sheets covering hen house roofs; 5) tires were used bicycle tires left outdoors within villages; 6) rocks holes were of irregular shapes, different sizes and were generally shallow, well exposed to solar radiation and located on lateritic carapaces; 7) storage containers were clay pots and plastic containers used to store potable water; 8) discarded containers were from human waste (broken clay pots, plastic bottles, bowls, metal box, used 


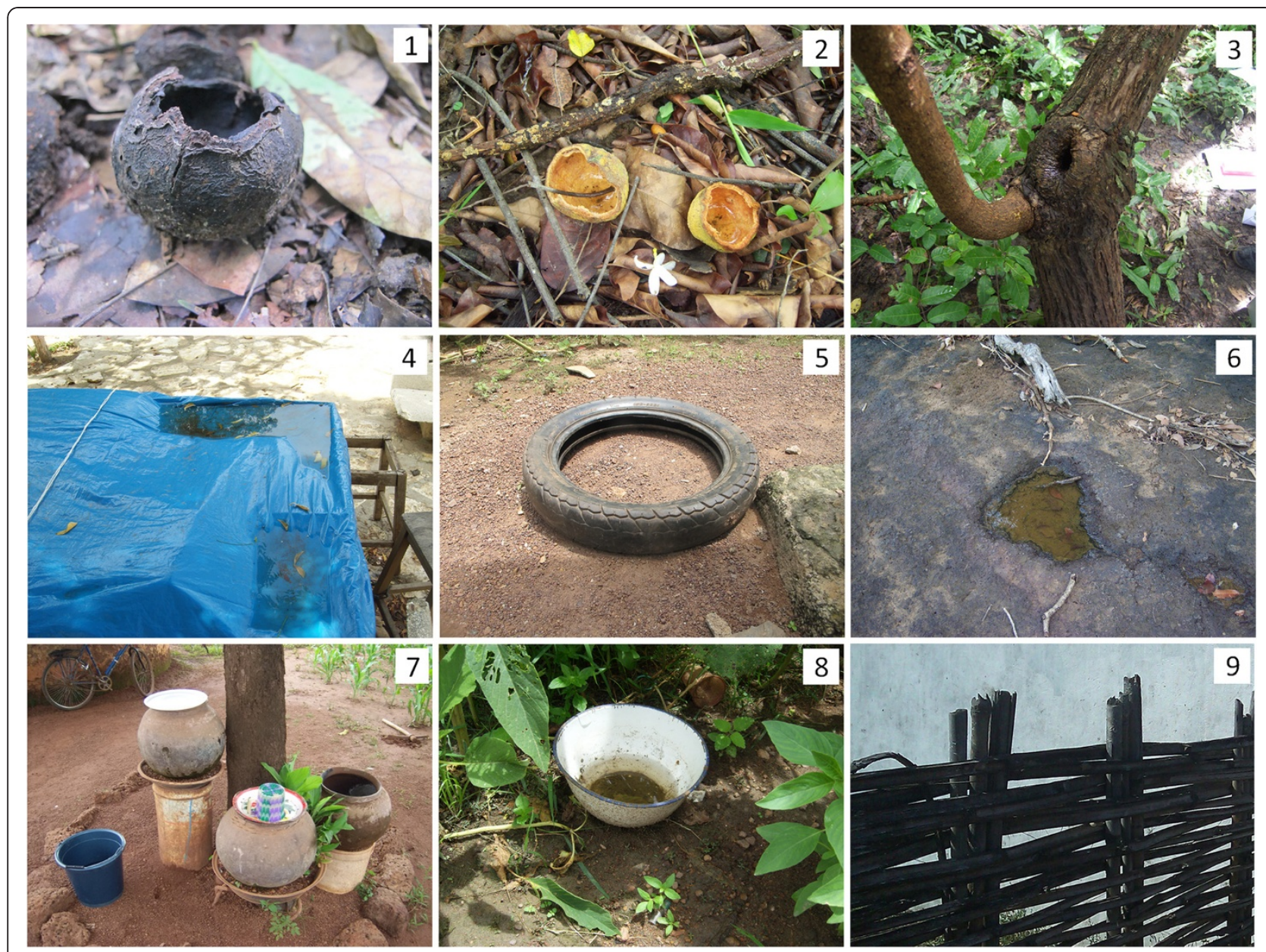

Figure 2 Immature mosquito microhabitats ( $1=$ decaying fruit husks, $2=$ fresh fruit husks, $3=$ tree holes, $4=$ puddles, $5=$ tires, $6=$ rocks-holes, $7=$ storage containers, $8=$ discarded containers and $9=$ bamboo holes) in Kédougou from June - December 2010.

cans, etc.) and 9) bamboo holes were cut bamboo used as fences within villages.

\section{Sampling procedure}

In each of the chosen blocks, the five land cover classes were surveyed and up to 30 of each of the 9 categorized microhabitats were examined for the presence of water and immature mosquitoes once per month from June, 2010 (just after the beginning of the rainy season) to January, 2011 (when no mosquito immature stages were found, thus the last mosquito larva was found in December, 2010). If larvae and/or pupae were present, the content of each hole was completely removed as follows. Small tree holes were emptied with a pipette of $10 \mathrm{ml}$ volume composed of a rigid plastic tube with a rubber suction bulb fitted to one end and/or with a mouth aspirator composed by a $150 \mathrm{ml}$ plastic pot with a cover connected to two flexible rubber tubes inserted through it. The longer one is inserted into the hole while the other is sucked for siphoning water out. Larger tree holes and rocks holes were sampled using either pipettes or small dippers (50-150 ml capacity). Bamboo holes were emptied by siphoning out the water with the mouth aspirator. Because all the larvae could not be removed by the initial siphoning, especially for tree holes [15], all holes were refilled with tap water and re-emptied until no larvae remained.

Hole contents were poured through a mesh net that retained all larval mosquito instars. The contents of discarded and storage containers were directly poured through a mesh net. For each sample (hole or container), the mesh net containing immature mosquitoes was submerged in tap water in a white plastic tray. Toxorhynchites spp. and Cx. tigripes larvae were removed from the sample to avoid predation of the other species. The content of the trays were placed in vials (different number depending on the quantity of larvae in the sample to avoid overcrowding and limit high mortalities of immature stages) labeled with a number corresponding to the microhabitat type, land 


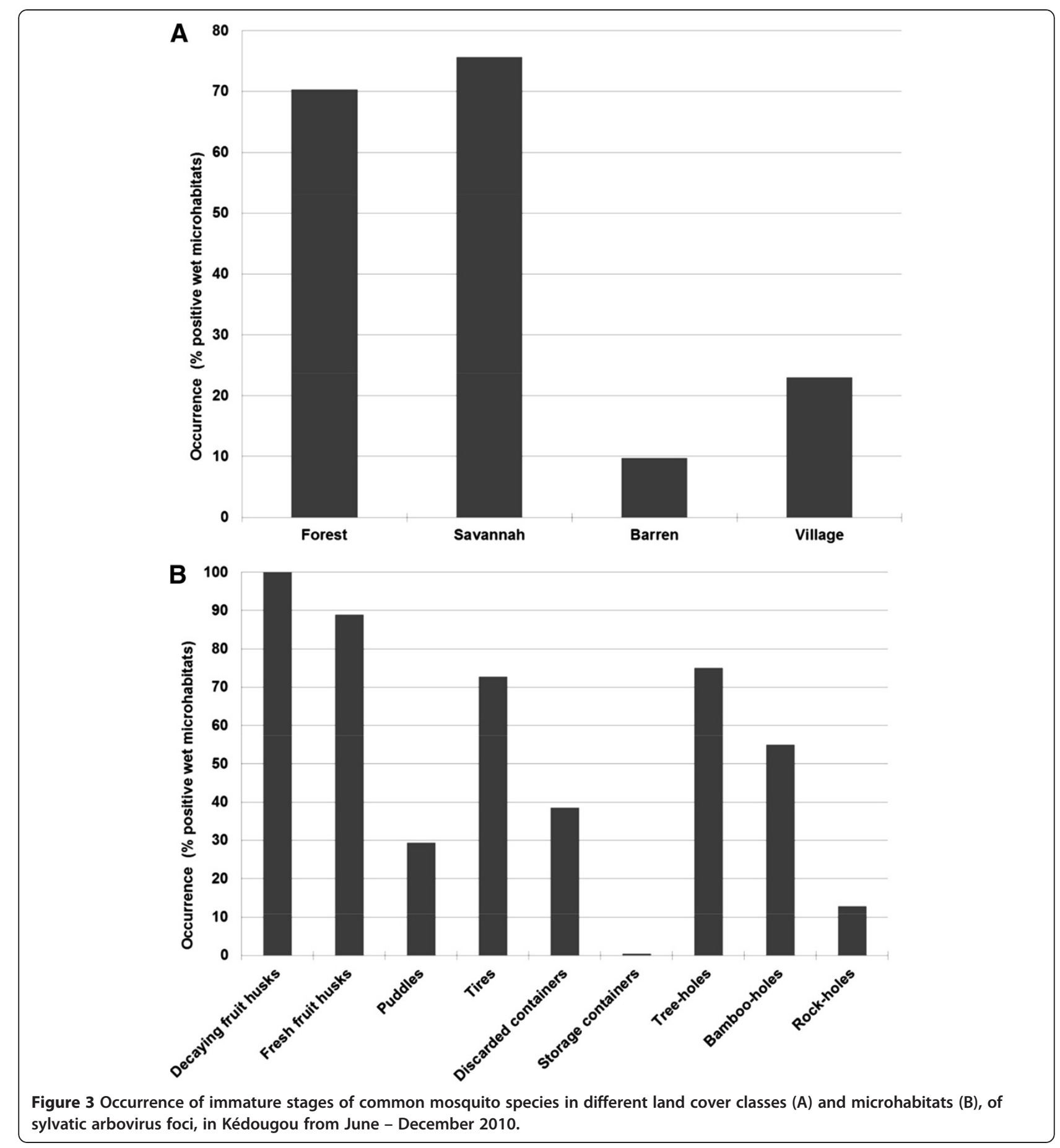

cover class and date of collection. The holes were refilled to their original volume with tap water. No volumetric record of the sizes of microhabitats was done. Immature stages were reared to adults in a field insectary, fed with larvae from a colony reared especially for that predacious species, and with TetraMin Baby Fish Food ${ }^{\circledR}$ for the others. Larval mortalities were relatively low and were not recorded. Only some tree holes and storage containers were repeatedly sampled on successive months. The other microhabitats were chosen randomly among all those available in the land covers.

Adults that emerged from larval collections were identified according to the keys of Edwards [16], Ferrara et al. [17], Huang [18] and Jupp [19] for the culicines and by Diagne et al. [20] for the anophelines. 


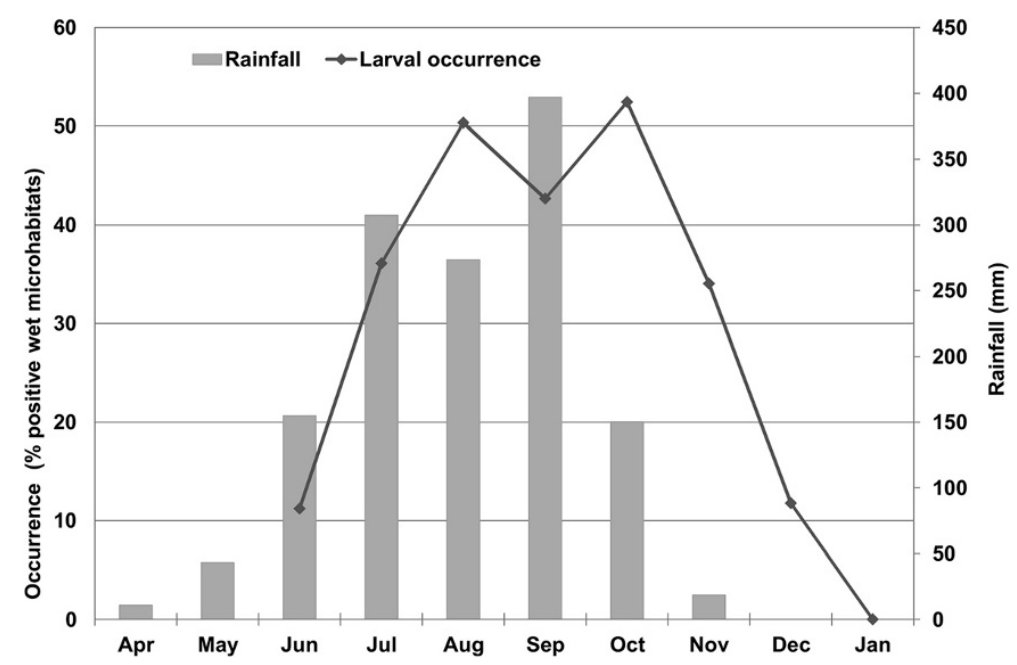

Figure 4 Seasonal occurrence of immature stages of common mosquito species in sylvatic arbovirus foci in Kédougou from June - December 2010.

\section{Data analysis}

Frequency of occurrence, expressed as the percentage of wet (water-holding) microhabitat that held immature mosquitoes, was calculated for the whole mosquito fauna and for each of the commonest species for each type of land cover class (macrohabitat) and microhabitat. Chi-square contingency tests were used to compare frequencies of occurrence of mosquitoes between types of micro and macrohabitats. Larval abundances (in the different micro and macrohabitats) were calculated as Williams' Means (Mw) [21]. The Kruskal-Wallis $H$ test was used to compare larval abundances between habitats and the Mann-Whitney $U$ test was used between pairs of habitats when the Kruskal-Wallis test was found to be statistically significant or when only two habitats were being compared. Differences were considered significant when $\mathrm{p}<0.05$. The number of species collected and the specific dominance were calculated and compared between habitats using the biodiversity module of Past $2.14^{\circledR}$. The $C_{7}$ index of Cole [22] was used to evaluate the interspecific associations between the species representing more than $1 \%$ of the total mosquito fauna collected, and the statistical significance tested with the corrected $X^{2}$ according Pielou [23]. The Fisher's exact test was used when one or more expected values were equal or less to five. All tests were conducted in StatView $5.0^{\circledR}$.

\section{Results}

\section{Habitat positivity}

Among the 2460 microhabitats examined, 1279 were wet, and $30.4 \%$ of these held at least one larva or pupa. Wet microhabitats were found in every land cover type except agricultural land. Larval occurrence (Figure 3) differed significantly among different land cover classes $\left(\mathrm{x}^{2}=370.7 ; \mathrm{df}=3 ; \mathrm{p}<0.0001\right)$ and microhabitats $\left(\mathrm{x}^{2}=\right.$ 549.6; df $=8 ; \mathrm{p}<0.0001)$. Microhabitats within forest and savannah land covers were most frequently occupied and were equally likely $\left(x^{2}=1.5 ; \mathrm{df}=1 ; \mathrm{p}=0.22\right)$ to be colonized by larvae of one or more species of mosquitoes. Regardless of the land cover type, immature mosquitoes were most often detected in decaying fruit husks, of which $100 \%$ contained at least one larva or pupa, followed by fresh fruit husks (89\%), tree holes (75\%) and tires (73\%). The lowest frequencies of immature mosquito occurrence were observed in rocks holes $(13 \%)$ and storage containers $(0.4 \%)$. The percentage of infested wet microhabitats increased from June (just after the first rains) and peaked twice between August and October, after which it decreased (Figure 4).

\section{Species composition}

Thirty-five species of mosquitoes in 7 genera were collected from natural and artificial water-holding microhabitats at our study sites from June to December, 2010 (Table 1). A total of 5121 mosquito adults emerged from larvae and pupae collected from forests (1858 specimens), savannahs (545), barren land (86) and villages (2632). Aedes aegypti formosus (the only sub species of Ae. aegypti present in the study area) was the dominant species, representing $30.2 \%$ of immature mosquitoes collected, followed by 12 other species, each comprising more than $1 \%$ of the mosquito fauna collected. The YFV, DENV-2 and CHIKV vectors included Ae. vittatus (7.9\%), Ae. luteocephalus (5.7\%), Ae. taylori (5.0\%) and Ae. furcifer (1.3\%). The 
Table 1 Total number of mosquitoes collected as larvae in different land cover classes, within foci of sylvatic arboviruses in Kédougou from June - December 2010

\begin{tabular}{|c|c|c|c|c|c|c|c|c|c|c|}
\hline \multirow[t]{2}{*}{ Species } & \multicolumn{2}{|c|}{ Forest } & \multicolumn{2}{|c|}{ Savannah } & \multicolumn{2}{|c|}{ Barren } & \multicolumn{2}{|c|}{ Village } & \multicolumn{2}{|c|}{ Total per species } \\
\hline & $\mathrm{Nb}$ & $\%$ & $\mathrm{Nb}$ & $\%$ & $\mathrm{Nb}$ & $\%$ & $\mathrm{Nb}$ & $\%$ & $\mathrm{Nb}$ & $\%$ \\
\hline Aedes aegypti & 108 & 5.8 & 29 & 5.3 & 8 & 9.3 & 1402 & 53.3 & 1547 & 30.2 \\
\hline Aedes africanus & 6 & 0.3 & & 0.0 & & 0.0 & & 0.0 & 6 & 0.1 \\
\hline Aedes argenteopunctatus & 1 & 0.1 & & 0.0 & & 0.0 & & 0.0 & 1 & 0.0 \\
\hline Aedes bromeliae & 4 & 0.2 & 1 & 0.2 & & 0.0 & 34 & 1.3 & 39 & 0.8 \\
\hline Aedes furcifer & 33 & 1.8 & 33 & 6.1 & & 0.0 & & 0.0 & 66 & 1.3 \\
\hline Aedes hirsutus & & 0.0 & & 0.0 & & 0.0 & 15 & 0.6 & 15 & 0.3 \\
\hline Aedes longipalpis & 99 & 5.3 & 6 & 1.1 & & 0.0 & 1 & 0.0 & 106 & 2.1 \\
\hline Aedes luteocephalus & 180 & 9.7 & 94 & 17.2 & & 0.0 & 18 & 0.7 & 292 & 5.7 \\
\hline Aedes metallicus & 1 & 0.1 & & 0.0 & & 0.0 & & 0.0 & 1 & 0.0 \\
\hline Aedes minutus & 1 & 0.1 & & 0.0 & & 0.0 & & 0.0 & 1 & 0.0 \\
\hline Aedes neoafricatus & 1 & 0.1 & 1 & 0.2 & & 0.0 & & 0.0 & 2 & 0.0 \\
\hline Aedes stokesi & 7 & 0.4 & & 0.0 & & 0.0 & & 0.0 & 7 & 0.1 \\
\hline Aedes taylori & 211 & 11.4 & 44 & 8.1 & & 0.0 & & 0.0 & 255 & 5.0 \\
\hline Aedes unilineatus & 51 & 2.7 & 40 & 7.3 & & 0.0 & 5 & 0.2 & 96 & 1.9 \\
\hline Aedes vittatus & 149 & 8.0 & 100 & 18.3 & 56 & 65.1 & 98 & 3.7 & 403 & 7.9 \\
\hline Anopheles coustani & 1 & 0.1 & & 0.0 & & 0.0 & & 0.0 & 1 & 0.0 \\
\hline Anopheles gambiae & 1 & 0.1 & & 0.0 & 1 & 1.2 & 7 & 0.3 & 9 & 0.2 \\
\hline Anopheles hancocki & & 0.0 & & 0.0 & 1 & 1.2 & & 0.0 & 1 & 0.0 \\
\hline Anopheles pretoriensis & 1 & 0.1 & & 0.0 & 1 & 1.2 & & 0.0 & 2 & 0.0 \\
\hline Anopheles rufipes & 2 & 0.1 & & 0.0 & 14 & 16.3 & & 0.0 & 16 & 0.3 \\
\hline Culex cinerus & 121 & 6.5 & 6 & 1.1 & & 0.0 & 39 & 1.5 & 166 & 3.2 \\
\hline Culex decens & 79 & 4.3 & 23 & 4.2 & & 0.0 & 12 & 0.5 & 114 & 2.2 \\
\hline Culex macfiei & 23 & 1.2 & 6 & 1.1 & & 0.0 & 2 & 0.1 & 31 & 0.6 \\
\hline Culex neavei & 1 & 0.1 & 1 & 0.2 & & 0.0 & & 0.0 & 2 & 0.0 \\
\hline Culex nebulosus & 238 & 12.8 & 37 & 6.8 & & 0.0 & 478 & 18.2 & 753 & 14.7 \\
\hline Culex perfuscus & 73 & 3.9 & 8 & 1.5 & 1 & 1.2 & 75 & 2.8 & 157 & 3.1 \\
\hline Culex tigripes & & 0.0 & 5 & 0.9 & & 0.0 & 33 & 1.3 & 38 & 0.7 \\
\hline Culex tritaeniorhynchus & 1 & 0.1 & 4 & 0.7 & 1 & 1.2 & 373 & 14.2 & 379 & 7.4 \\
\hline Eretmapodites chrysogaster & 401 & 21.6 & 99 & 18.2 & 3 & 3.5 & 26 & 1.0 & 529 & 10.3 \\
\hline Eretmapodites oedipodius & & 0.0 & & 0.0 & & 0.0 & 1 & 0.0 & 1 & 0.0 \\
\hline Eretmapodites quinquevittatus & 4 & 0.2 & 2 & 0.4 & & 0.0 & 10 & 0.4 & 16 & 0.3 \\
\hline Ficalbia circumtestea & 10 & 0.5 & & 0.0 & & 0.0 & & 0.0 & 10 & 0.2 \\
\hline Toxorhynchites brevipalpis & 24 & 1.3 & 4 & 0.7 & & 0.0 & 3 & 0.1 & 31 & 0.6 \\
\hline Toxorhynchites lutescens & 3 & 0.2 & 2 & 0.4 & & 0.0 & & 0.0 & 5 & 0.1 \\
\hline Uranotaenia mashonensis & 23 & 1.2 & & 0.0 & & 0.0 & & 0.0 & 23 & 0.4 \\
\hline Total per land cover & 1858 & 100 & 545 & 100 & 86 & 100 & 2632 & 100 & 5121 & 100 \\
\hline
\end{tabular}

dominant species varied according to the land cover and microhabitat investigated and was Eretmapodites chrysogaster $(21.6 \%)$ in the forest, Ae. vittatus in the savannah (18.3\%) and barren (65.1\%) and Ae aegypti in the village (53.3\%). Among microhabitats (Table 2), the dominant species were Ae. aegypti in the storage containers (100\% of the collected fauna), tires (96\%), bamboo holes (90.6\%) and discarded containers (51.4\%), Er. chrysogaster in fresh fruit husks (64.7\%) and decaying fruit husks (48.4\%), Ae. vittatus in puddles (52.3) and rocks holes (48.3) and finally Ae. luteocephalus in tree holes with $20.2 \%$ of the collected fauna. 
Table 2 Mosquitoes collected as larvae in different microhabitats, in sylvatic arbovirus foci in Kédougou from June - December 2010

\begin{tabular}{|c|c|c|c|c|c|c|c|c|c|c|c|c|c|c|c|c|c|c|}
\hline \multirow[t]{2}{*}{ Species } & \multicolumn{2}{|c|}{$\begin{array}{c}\text { Bamboo } \\
\text { holes }\end{array}$} & \multicolumn{2}{|c|}{$\begin{array}{l}\text { Discarded } \\
\text { containers }\end{array}$} & \multicolumn{2}{|c|}{$\begin{array}{l}\text { Decaying } \\
\text { fruit husks }\end{array}$} & \multicolumn{2}{|c|}{$\begin{array}{c}\text { Fresh } \\
\text { fruit husks }\end{array}$} & \multicolumn{2}{|c|}{ Puddles } & \multicolumn{2}{|c|}{$\begin{array}{l}\text { Rock } \\
\text { holes }\end{array}$} & \multicolumn{2}{|c|}{$\begin{array}{c}\text { Storage } \\
\text { containers }\end{array}$} & \multicolumn{2}{|c|}{$\begin{array}{l}\text { Tree } \\
\text { holes }\end{array}$} & \multicolumn{2}{|c|}{ Tires } \\
\hline & $\overline{\mathrm{Nb}}$ & $\%$ & $\mathrm{Nb}$ & $\%$ & $\mathrm{Nb}$ & $\%$ & $\mathrm{Nb}$ & $\%$ & $\mathrm{Nb}$ & $\%$ & $\mathrm{Nb}$ & $\%$ & $\mathrm{Nb}$ & $\%$ & $\mathrm{Nb}$ & $\%$ & $\mathrm{Nb}$ & $\%$ \\
\hline Aedes aegypti & 48 & 90.6 & 1206 & 51.4 & 49 & 38.9 & 57 & 8.7 & 27 & 11.1 & 36 & 8.9 & 97 & 100 & 84 & 6.6 & 24 & 96 \\
\hline Aedes africanus & 0 & 0.0 & 0 & 0.0 & 0 & 0.0 & 0 & 0.0 & 0 & 0.0 & 0 & 0.0 & 0 & 0 & 6 & 0.5 & 0 & 0 \\
\hline Aedes argenteopunctatus & 0 & 0.0 & 0 & 0.0 & 0 & 0.0 & 0 & 0.0 & 0 & 0.0 & 0 & 0.0 & 0 & 0 & 1 & 0.1 & 0 & 0 \\
\hline Aedes bromeliae & 0 & 0.0 & 31 & 1.3 & 1 & 0.8 & 2 & 0.3 & 0 & 0.0 & 0 & 0.0 & 0 & 0 & 2 & 0.2 & 1 & 4 \\
\hline Aedes furcifer & 0 & 0.0 & 0 & 0.0 & 0 & 0.0 & 3 & 0.5 & 0 & 0.0 & 0 & 0.0 & 0 & 0 & 63 & 5.0 & 0 & 0 \\
\hline Aedes hirsutus & 0 & 0.0 & 9 & 0.4 & 0 & 0.0 & 0 & 0.0 & 6 & 2.5 & 0 & 0.0 & 0 & 0 & 0 & 0.0 & 0 & 0 \\
\hline Aedes longipalpis & 0 & 0.0 & 0 & 0.0 & 0 & 0.0 & 1 & 0.2 & 1 & 0.4 & 0 & 0.0 & 0 & 0 & 104 & 8.2 & 0 & 0 \\
\hline Aedes luteocephalus & 0 & 0.0 & 14 & 0.6 & 3 & 2.4 & 16 & 2.4 & 3 & 1.2 & 0 & 0.0 & 0 & 0 & 256 & 20.2 & 0 & 0 \\
\hline Aedes metallicus & 0 & 0.0 & 0 & 0.0 & 0 & 0.0 & 0 & 0.0 & 0 & 0.0 & 0 & 0.0 & 0 & 0 & 1 & 0.1 & 0 & 0 \\
\hline Aedes minutus & 0 & 0.0 & 0 & 0.0 & 0 & 0.0 & 0 & 0.0 & 0 & 0.0 & 0 & 0.0 & 0 & 0 & 1 & 0.1 & 0 & 0 \\
\hline Aedes neoafricatus & 0 & 0.0 & 0 & 0.0 & 0 & 0.0 & 0 & 0.0 & 0 & 0.0 & 0 & 0.0 & 0 & 0 & 2 & 0.2 & 0 & 0 \\
\hline Aedes stokesi & 0 & 0.0 & 0 & 0.0 & 0 & 0.0 & 0 & 0.0 & 0 & 0.0 & 0 & 0.0 & 0 & 0 & 7 & 0.6 & 0 & 0 \\
\hline Aedes taylori & 0 & 0.0 & 0 & 0.0 & 0 & 0.0 & 2 & 0.3 & 0 & 0.0 & 0 & 0.0 & 0 & 0 & 253 & 20.0 & 0 & 0 \\
\hline Aedes unilineatus & 3 & 5.7 & 2 & 0.1 & 5 & 4.0 & 19 & 2.9 & 0 & 0.0 & 1 & 0.2 & 0 & 0 & 66 & 5.2 & 0 & 0 \\
\hline Aedes vittatus & 0 & 0.0 & 69 & 2.9 & 0 & 0.0 & 3 & 0.5 & 127 & 52.3 & 195 & 48.3 & 0 & 0 & 9 & 0.7 & 0 & 0 \\
\hline Anopheles coustani & 0 & 0.0 & 0 & 0.0 & 0 & 0.0 & 1 & 0.2 & 0 & 0.0 & 0 & 0.0 & 0 & 0 & 0 & 0.0 & 0 & 0 \\
\hline Anopheles gambiae & 0 & 0.0 & 7 & 0.3 & 0 & 0.0 & 0 & 0.0 & 0 & 0.0 & 2 & 0.5 & 0 & 0 & 0 & 0.0 & 0 & 0 \\
\hline Anopheles hancocki & 0 & 0.0 & 0 & 0.0 & 0 & 0.0 & 0 & 0.0 & 0 & 0.0 & 1 & 0.2 & 0 & 0 & 0 & 0.0 & 0 & 0 \\
\hline Anopheles pretoriensis & 0 & 0.0 & 0 & 0.0 & 0 & 0.0 & 0 & 0.0 & 0 & 0.0 & 3 & 0.7 & 0 & 0 & 0 & 0.0 & 0 & 0 \\
\hline Anopheles rufipes & 0 & 0.0 & 0 & 0.0 & 0 & 0.0 & 0 & 0.0 & 0 & 0.0 & 14 & 3.5 & 0 & 0 & 2 & 0.2 & 0 & 0 \\
\hline Culex cinerus & 0 & 0.0 & 33 & 1.4 & 1 & 0.8 & 10 & 1.5 & 6 & 2.5 & 0 & 0.0 & 0 & 0 & 116 & 9.2 & 0 & 0 \\
\hline Culex decens & 0 & 0.0 & 11 & 0.5 & 0 & 0.0 & 1 & 0.2 & 12 & 4.9 & 74 & 18.3 & 0 & 0 & 16 & 1.3 & 0 & 0 \\
\hline Culex macfiei & 0 & 0.0 & 2 & 0.1 & 0 & 0.0 & 1 & 0.2 & 0 & 0.0 & 4 & 1.0 & 0 & 0 & 27 & 2.1 & 0 & 0 \\
\hline Culex neavei & 0 & 0.0 & 0 & 0.0 & 0 & 0.0 & 0 & 0.0 & 0 & 0.0 & 1 & 0.2 & 0 & 0 & 1 & 0.1 & 0 & 0 \\
\hline Culex nebulosus & 0 & 0.0 & 485 & 20.7 & 2 & 1.6 & 106 & 16.2 & 19 & 7.8 & 6 & 1.5 & 0 & 0 & 140 & 11.1 & 0 & 0 \\
\hline Culex perfuscus & 0 & 0.0 & 53 & 2.3 & 0 & 0.0 & 1 & 0.2 & 23 & 9.5 & 37 & 9.2 & 0 & 0 & 43 & 3.4 & 0 & 0 \\
\hline Culex tigripes & 0 & 0.0 & 32 & 1.4 & 0 & 0.0 & 0 & 0.0 & 1 & 0.4 & 0 & 0.0 & 0 & 0 & 5 & 0.4 & 0 & 0 \\
\hline Culex tritaeniorhynchus & 0 & 0.0 & 355 & 15.1 & 2 & 1.6 & 2 & 0.3 & 18 & 7.4 & 3 & 0.7 & 0 & 0 & 1 & 0.1 & 0 & 0 \\
\hline Eretmapodites chrysogaster & 2 & 3.8 & 24 & 1.0 & 61 & 48.4 & 424 & 64.7 & 0 & 0.0 & 4 & 1.0 & 0 & 0 & 14 & 1.1 & 0 & 0 \\
\hline Eretmapodites oedipodius & 0 & 0.0 & 1 & 0.0 & 0 & 0.0 & 0 & 0.0 & 0 & 0.0 & 0 & 0.0 & 0 & 0 & 0 & 0.0 & 0 & 0 \\
\hline Eretmapodites quinquevittatus & 0 & 0.0 & 10 & 0.4 & 2 & 1.6 & 3 & 0.5 & 0 & 0.0 & 0 & 0.0 & 0 & 0 & 1 & 0.1 & 0 & 0 \\
\hline Ficalbia circumtestea & 0 & 0.0 & 0 & 0.0 & 0 & 0.0 & 0 & 0.0 & 0 & 0.0 & 0 & 0.0 & 0 & 0 & 10 & 0.8 & 0 & 0 \\
\hline Toxorhynchites brevipalpis & 0 & 0.0 & 1 & 0.0 & 0 & 0.0 & 2 & 0.3 & 0 & 0.0 & 0 & 0.0 & 0 & 0 & 29 & 2.3 & 0 & 0 \\
\hline Toxorhynchites lutescens & 0 & 0.0 & 0 & 0.0 & 0 & 0.0 & 1 & 0.2 & 0 & 0.0 & 0 & 0.0 & 0 & 0 & 6 & 0.5 & 0 & 0 \\
\hline Uranotaenia mashonensis & 0 & 0.0 & 0 & 0.0 & 0 & 0.0 & 0 & 0.0 & 0 & 0.0 & 23 & 5.7 & 0 & 0 & 0 & 0.0 & 0 & 0 \\
\hline Total per microhabitat & 53 & 100 & 2345 & 100 & 126 & 100 & 655 & 100 & 243 & 100 & 404 & 100 & 97 & 100 & 1266 & 100 & 25 & 100 \\
\hline
\end{tabular}


Table 3 Seasonal occurrence (\% of positive wet containers) of immature stages of common mosquito species in different land covers and microhabitats, foci of sylvatic arbovirus, in Kédougou from June - December 2010

\begin{tabular}{|c|c|c|c|c|c|c|c|c|c|}
\hline Land covers & Species & Jun & Jul & Aug & Sep & Oct & Nov & Dec & Mean \\
\hline \multirow[t]{13}{*}{ Forest } & Aedes aegypti & $10.8(4)$ & $16.4(9)$ & $10.3(9)$ & $3.3(3)$ & $4.8(2)$ & $14.3(2)$ & $0(0)$ & $8.7(29)$ \\
\hline & Aedes furcifer & $5.4(2)$ & $7.3(4)$ & $7.7(6)$ & $6.7(6)$ & $2.4(1)$ & $0(0)$ & $0(0)$ & $3.9(13)$ \\
\hline & Aedes longipalpis & $10.8(4)$ & $10.9(6)$ & $10.2(8)$ & $4.4(4)$ & $7.1(3)$ & $0(0)$ & $0(0)$ & $7.5(25)$ \\
\hline & Aedes luteocephalus & $16.2(6)$ & $21.8(12)$ & $9.0(7)$ & $8.9(8)$ & $7.1(3)$ & $0(0)$ & $5.9(1)$ & $10.8(36)$ \\
\hline & Aedes taylori & $16.2(6)$ & $25.4(14)$ & $7.7(6)$ & $7.8(7)$ & $2.4(1)$ & $0(0)$ & $0(0)$ & $10.2(34)$ \\
\hline & Aedes unilineatus & $13.5(5)$ & $10.9(6)$ & $5.1(4)$ & $5.6(5)$ & $0(0)$ & $7.1(1)$ & $5.9(1)$ & $6.3(21)$ \\
\hline & Aedes vittatus & $13.5(5)$ & $3.6(2)$ & $6.4(5)$ & $1.1(1)$ & $4.8(2)$ & $14.3(2)$ & $0(0)$ & $5.1(17)$ \\
\hline & Culex cinerus & $13.5(5)$ & $36.4(20)$ & $5.1(4)$ & $0(0)$ & $0(0)$ & $0(0)$ & $0(0)$ & $8.7(29)$ \\
\hline & Culex decens & $5.4(2)$ & $1.8(1)$ & $3.8(3)$ & $1.1(1)$ & $9.5(4)$ & $0(0)$ & $0(0)$ & $3.3(11)$ \\
\hline & Culex nebulosus & $16.2(6)$ & $27.3(15)$ & $10.2(8)$ & $2.2(2)$ & $0(0)$ & $7.1(1)$ & $5.9(1)$ & $9.6(32)$ \\
\hline & Culex perfuscus & $5.4(2)$ & $1.8(1)$ & $0(0)$ & $1.1(1)$ & $9.5(4)$ & $14.3(2)$ & $0(0)$ & $3.0(10)$ \\
\hline & Culex tritaeniorhynchus & $0(0)$ & $0(0)$ & $0(0)$ & $0(0)$ & $2.4(1)$ & $0(0)$ & $0(0)$ & $0.3(1)$ \\
\hline & Eretmapodites chrysogaster & $2.7(1)$ & $14.5(8)$ & $46.1(36)$ & $15.5(14)$ & $16.7(7)$ & $0(0)$ & $0(0)$ & $19.8(66)$ \\
\hline \multirow[t]{13}{*}{ Savannah } & Aedes aegypti & $0(0)$ & $24(6)$ & $29.5(13)$ & $10.7(7)$ & $11.1(1)$ & na & $0(0)$ & $18.2(27)$ \\
\hline & Aedes furcifer & $0(0)$ & $4(1)$ & $15.9(7)$ & $10.7(7)$ & $11.1(1)$ & na & $0(0)$ & $10.8(16)$ \\
\hline & Aedes longipalpis & $0(0)$ & $0(0)$ & $2.3(1)$ & $1.5(1)$ & $0(0)$ & na & $0(0)$ & $1.4(2)$ \\
\hline & Aedes luteocephalus & $0(0)$ & $12(3)$ & $18.2(8)$ & $17.9(12)$ & $22.2(2)$ & na & $0(0)$ & $16.9(25)$ \\
\hline & Aedes taylori & $0(0)$ & $12(3)$ & $15.9(7)$ & $6.0(4)$ & $0(0)$ & na & $0(0)$ & $9.5(14)$ \\
\hline & Aedes unilineatus & $0(0)$ & $8(2)$ & $13.6(6)$ & $9.0(6)$ & $11.1(1)$ & na & $0(0)$ & $10.1(15)$ \\
\hline & Aedes vittatus & $50(1)$ & $4(1)$ & $4.5(2)$ & $0(0)$ & $0(0)$ & na & $0(0)$ & $2.7(4)$ \\
\hline & Culex cinerus & $0(0)$ & $4(1)$ & $4.5(2)$ & $0(0)$ & $0(0)$ & na & $0(0)$ & $2.0(3)$ \\
\hline & Culex decens & $0(0)$ & $0(0)$ & $9.1(4)$ & $0(0)$ & $0(0)$ & na & $0(0)$ & $2.7(4)$ \\
\hline & Culex nebulosus & $0(0)$ & $20(5)$ & $9.1(4)$ & $1.5(1)$ & $0(0)$ & na & $0(0)$ & $6.8(10)$ \\
\hline & Culex perfuscus & $0(0)$ & $0(0)$ & $6.8(3)$ & $3.0(2)$ & $0(0)$ & na & $0(0)$ & $3.4(5)$ \\
\hline & Culex tritaeniorhynchus & $0(0)$ & $4(1)$ & $0(0)$ & $1.5(1)$ & $0(0)$ & na & $0(0)$ & $1.4(2)$ \\
\hline & Eretmapodites chrysogaster & $0(0)$ & $24(6)$ & $36.4(16)$ & $9.0(6)$ & $0(0)$ & na & $0(0)$ & $18.9(28)$ \\
\hline \multirow[t]{6}{*}{ Barren } & Aedes aegypti & $0(0)$ & $1.7(1)$ & $0(0)$ & $0(0)$ & $0(0)$ & na & na & $0.4(1)$ \\
\hline & Aedes vittatus & $0(0)$ & $6.7(4)$ & $5.0(3)$ & $1.7(1)$ & $8.0(2)$ & na & na & $4.3(10)$ \\
\hline & Culex nebulosus & $0(0)$ & $1.7(1)$ & $1.7(1)$ & $0(0)$ & $0(0)$ & na & na & $0.9(2)$ \\
\hline & Culex perfuscus & $0(0)$ & $0(0)$ & $0(0)$ & $0(0)$ & $4.0(1)$ & na & na & $0.4(1)$ \\
\hline & Culex tritaeniorhynchus & $0(0)$ & $1.7(1)$ & $0(0)$ & $0(0)$ & $8.0(2)$ & na & na & $1.3(3)$ \\
\hline & Eretmapodites chrysogaster & $0(0)$ & $0(0)$ & $0(0)$ & $0(0)$ & $4.0(1)$ & na & na & $0.4(1)$ \\
\hline \multirow[t]{11}{*}{ Village } & Aedes aegypti & $5.1(4)$ & $7.8(7)$ & $23.1(25)$ & $26.0(32)$ & $11.9(8)$ & $0(0)$ & $0(0)$ & $13.9(76)$ \\
\hline & Aedes longipalpis & $1.3(1)$ & $0(0)$ & $0(0)$ & $0(0)$ & $0(0)$ & $0(0)$ & $0(0)$ & $0.2(1)$ \\
\hline & Aedes luteocephalus & $0(0)$ & $4.4(4)$ & $1.8(2)$ & $4.1(5)$ & $1.5(1)$ & $0(0)$ & $0(0)$ & $2.2(12)$ \\
\hline & Aedes unilineatus & $0(0)$ & $0(0)$ & $0.9(1)$ & $2.4(3)$ & $0(0)$ & $0(0)$ & $0(0)$ & $0.7(4)$ \\
\hline & Aedes vittatus & $2.5(2)$ & $3.3(3)$ & $4.6(5)$ & $0.8(1)$ & $1.5(1)$ & $0(0)$ & $0(0)$ & $2.2(12)$ \\
\hline & Culex cinerus & $2.5(2)$ & $2.2(2)$ & $2.8(3)$ & $0(0)$ & $0(0)$ & $0(0)$ & $0(0)$ & $1.3(7)$ \\
\hline & Culex decens & $0(0)$ & $1.1(1)$ & $1.8(2)$ & $1.6(2)$ & $0(0)$ & $0(0)$ & $0(0)$ & $0.9(5)$ \\
\hline & Culex nebulosus & $3.8(3)$ & $3.3(3)$ & $5.5(6)$ & $3.2(4)$ & $4.5(3)$ & $2.8(1)$ & $2.3(1)$ & $3.9(21)$ \\
\hline & Culex perfuscus & $1.3(1)$ & $3.3(3)$ & $3.7(4)$ & $0(0)$ & $1.5(1)$ & $0(0)$ & $0(0)$ & $1.7(9)$ \\
\hline & Culex tritaeniorhynchus & $0(0)$ & $12.2(11)$ & $2.8(3)$ & $5.7(7)$ & $4.5(3)$ & $2.8(1)$ & $0(0)$ & $4.6(25)$ \\
\hline & Eretmapodites chrysogaster & $0(0)$ & $0(0)$ & $1.7(1)$ & $2.4(3)$ & $4.5(3)$ & $0(0)$ & $2.3(1)$ & $1.5(8)$ \\
\hline
\end{tabular}


Table 3 Seasonal occurrence (\% of positive wet containers) of immature stages of common mosquito species in different land covers and microhabitats, foci of sylvatic arbovirus, in Kédougou from June - December 2010 (Continued)

\begin{tabular}{|c|c|c|c|c|c|c|c|c|c|}
\hline \multicolumn{10}{|l|}{ Microhabitat } \\
\hline \multirow[t]{7}{*}{ Decaying fruit husks } & Aedes aegypti & na & $60.7(4)$ & $33.3(3)$ & na & na & na & na & $46.7(7)$ \\
\hline & Aedes luteocephalus & na & $0.0(0)$ & $11.1(1)$ & na & na & na & na & $6.7(1)$ \\
\hline & Aedes unilineatus & na & $16.7(1)$ & $33.3(3)$ & na & na & na & na & $26.7(4)$ \\
\hline & Culex cinerus & na & $16.7(1)$ & $0.0(0)$ & na & na & na & na & $6.7(1)$ \\
\hline & Culex nebulosus & na & $33.3(2)$ & $0.0(0)$ & na & na & na & na & $13.3(2)$ \\
\hline & Culex tritaeniorhynchus & na & $16.7(1)$ & $0.0(0)$ & na & na & na & na & $6.7(1)$ \\
\hline & Eretmapodites chrysogaster & na & $50.0(3)$ & $100(9)$ & na & na & na & na & $80.0(12)$ \\
\hline \multirow[t]{13}{*}{ Fresh fruit husks } & Aedes aegypti & $0.0(0)$ & $22.2(4)$ & $30.9(13)$ & $2.1(1)$ & $6.7(1)$ & na & na & $15.0(19)$ \\
\hline & Aedes furcifer & $0.0(0)$ & $5.6(1)$ & $2.4(1)$ & $2.1(1)$ & $0.0(0)$ & na & na & $2.4(3)$ \\
\hline & Aedes longipalpis & $0.0(0)$ & $0.0(0)$ & $2.4(1)$ & $0.0(0)$ & $0.0(0)$ & na & na & $0.8(1)$ \\
\hline & Aedes luteocephalus & $0.0(0)$ & $0.0(0)$ & $4.8(2)$ & $4.2(2)$ & $6.7(1)$ & na & na & $3.9(5)$ \\
\hline & Aedes taylori & $0.0(0)$ & $5.6(1)$ & $0.0(0)$ & $2.1(1)$ & $0.0(0)$ & na & na & $1.6(2)$ \\
\hline & Aedes unilineatus & $0.0(0)$ & $0.0(0)$ & $7.1(3)$ & $4.2(2)$ & $0.0(0)$ & na & na & $3.9(5)$ \\
\hline & Aedes vittatus & $0.0(0)$ & $0.0(0)$ & $4.8(2)$ & $0.0(0)$ & $0.0(0)$ & na & na & $1.6(2)$ \\
\hline & Culex cinerus & $0.0(0)$ & $16.7(3)$ & $0.0(0)$ & $0.0(0)$ & $0.0(0)$ & na & na & $2.4(3)$ \\
\hline & Culex decens & $0.0(0)$ & $0.0(0)$ & $2.4(1)$ & $0.0(0)$ & $0.0(0)$ & na & na & $0.8(1)$ \\
\hline & Culex nebulosus & $0.0(0)$ & $27.8(5)$ & $4.8(2)$ & $0.0(0)$ & $0.0(0)$ & na & na & $5.5(7)$ \\
\hline & Culex perfuscus & $0.0(0)$ & $0.0(0)$ & $0.0(0)$ & $0.0(0)$ & $6.7(1)$ & na & na & $0.8(1)$ \\
\hline & Culex tritaeniorhynchus & $0.0(0)$ & $0.0(0)$ & $0.0(0)$ & $2.1(1)$ & $0.0(0)$ & na & na & $0.8(1)$ \\
\hline & Eretmapodites chrysogaster & $0.0(0)$ & $55.6(10)$ & $92.8(39)$ & $38.3(18)$ & $40(6)$ & na & na & $57.5(73)$ \\
\hline \multirow[t]{9}{*}{ Puddles } & Aedes aegypti & $16.7(1)$ & $22.2(2)$ & $18.2(2)$ & $0(0)$ & $100(1)$ & na & $0.0(0)$ & $11.8(6)$ \\
\hline & Aedes longipalpis & $16.7(1)$ & $0.0(0)$ & $0.0(0)$ & $0.0(0)$ & $0.0(0)$ & na & $0.0(0)$ & $2.0(1)$ \\
\hline & Aedes luteocephalus & $0.0(0)$ & $11.1(1)$ & $9.1(1)$ & $4.8(1)$ & $0.0(0)$ & na & $0.0(0)$ & $5.9(3)$ \\
\hline & Aedes vittatus & $16.7(1)$ & $33.3(3)$ & $27.3(3)$ & $0.0(0)$ & $0.0(0)$ & na & $0.0(0)$ & $13.7(7)$ \\
\hline & Culex cinerus & $16.7(1)$ & $11.1(1)$ & $9.1(1)$ & $0.0(0)$ & $0.0(0)$ & na & $0.0(0)$ & $5.9(3)$ \\
\hline & Culex decens & $0.0(0)$ & $0.0(0)$ & $9.1(1)$ & $4.8(1)$ & $0.0(0)$ & na & $0.0(0)$ & $3.9(2)$ \\
\hline & Culex nebulosus & $16.7(1)$ & $33.3(3)$ & $9.1(1)$ & $0.0(0)$ & $100(1)$ & na & $0.0(0)$ & $11.8(6)$ \\
\hline & Culex perfuscus & $0.0(0)$ & $22.2(2)$ & $9.1(1)$ & $4.8(1)$ & $0.0(0)$ & na & $0.0(0)$ & $7.8(4)$ \\
\hline & Culex tritaeniorhynchus & $0.0(0)$ & $33.3(3)$ & $0.0(0)$ & $0.0(0)$ & $0.0(0)$ & na & $0.0(0)$ & $5.9(3)$ \\
\hline \multirow[t]{10}{*}{ Discarded containers } & Aedes aegypti & $7.3(3)$ & $9.7(4)$ & $38.5(20)$ & $37.1(23)$ & $21.9(7)$ & $0.0(0)$ & $0.0(0)$ & $23.7(57)$ \\
\hline & Aedes luteocephalus & $0.0(0)$ & $7.3(3)$ & $1.9(1)$ & $4.8(3)$ & $3.1(1)$ & $0.0(0)$ & $10(1)$ & $3.7(9)$ \\
\hline & Aedes unilineatus & $0.0(0)$ & $0.0(0)$ & $1.9(1)$ & $1.6(1)$ & $0.0(0)$ & $0.0(0)$ & $0.0(0)$ & $0.8(2)$ \\
\hline & Aedes vittatus & $2.4(1)$ & $2.4(1)$ & $5.8(3)$ & $1.6(1)$ & $3.1(1)$ & $0.0(0)$ & $0.0(0)$ & $2.9(7)$ \\
\hline & Culex cinerus & $2.4(1)$ & $2.4(1)$ & $3.8(2)$ & $0.0(0)$ & $0.0(0)$ & $0.0(0)$ & $0.0(0)$ & $1.7(4)$ \\
\hline & Culex decens & $0.0(0)$ & $2.4(1)$ & $1.9(1)$ & $3.2(2)$ & $0.0(0)$ & $0.0(0)$ & $0.0(0)$ & $1.7(4)$ \\
\hline & Culex nebulosus & $4.9(2)$ & $2.4(1)$ & $9.6(5)$ & $4.8(3)$ & $6.2(2)$ & $33.3(1)$ & $20(2)$ & $6.6(16)$ \\
\hline & Culex perfuscus & $2.4(1)$ & $2.4(1)$ & $5.8(3)$ & $0.0(0)$ & $3.1(1)$ & $0.0(0)$ & $10(1)$ & $2.9(7)$ \\
\hline & Culex tritaeniorhynchus & $0.0(0)$ & $19.5(8)$ & $5.8(3)$ & $11.3(7)$ & $9.4(3)$ & $33.3(1)$ & $0.0(0)$ & $9.1(22)$ \\
\hline & Eretmapodites chrysogaster & $0.0(0)$ & $0.0(0)$ & $1.9(1)$ & $4.8(3)$ & $6.2(2)$ & $0.0(0)$ & $10(1)$ & $2.9(7)$ \\
\hline
\end{tabular}


Table 3 Seasonal occurrence (\% of positive wet containers) of immature stages of common mosquito species in different land covers and microhabitats, foci of sylvatic arbovirus, in Kédougou from June - December 2010 (Continued)

\begin{tabular}{|c|c|c|c|c|c|c|c|c|c|}
\hline Storage containers & Aedes aegypti & $0.0(0)$ & $2.5(1)$ & $0.0(0)$ & $0.0(0)$ & $0.0(0)$ & $0.0(0)$ & $0.0(0)$ & $0.4(1)$ \\
\hline \multirow[t]{13}{*}{ Tree holes } & Aedes aegypti & $17.4(4)$ & $15.5(7)$ & $8.2(5)$ & $11.6(10)$ & $6.7(2)$ & $0.0(0)$ & $0.0(0)$ & $10.9(28)$ \\
\hline & Aedes furcifer & $8.7(2)$ & $8.9(4)$ & $19.7(12)$ & $13.9(12)$ & $6.7(2)$ & $0.0(0)$ & $0.0(0)$ & $12.5(32)$ \\
\hline & Aedes longipalpis & $17.4(4)$ & $13.3(6)$ & $13.1(8)$ & $5.8(5)$ & $10(3)$ & $0.0(0)$ & $0.0(0)$ & $10.2(26)$ \\
\hline & Aedes luteocephalus & $26.1(6)$ & $33.3(15)$ & $19.7(12)$ & $22.1(19)$ & $13.3(4)$ & $0.0(0)$ & $0.0(0)$ & $21.9(56)$ \\
\hline & Aedes taylori & $26.1(6)$ & $35.5(16)$ & $21.3(13)$ & $11.6(10)$ & $3.3(1)$ & $0.0(0)$ & $0.0(0)$ & $18.0(46)$ \\
\hline & Aedes unilineatus & $17.4(4)$ & $15.5(7)$ & $6.5(4)$ & $10.5(9)$ & $3.3(1)$ & $25(1)$ & $14.3(1)$ & $10.5(27)$ \\
\hline & Aedes vittatus & $13.0(3)$ & $2.2(1)$ & $3.3(2)$ & $1.2(1)$ & $3.3(1)$ & $0.0(0)$ & $0.0(0)$ & $3.1(8)$ \\
\hline & Culex cinerus & $21.7(5)$ & $37.8(17)$ & $9.8(6)$ & $0.0(0)$ & $0.0(0)$ & $0.0(0)$ & $0.0(0)$ & $10.9(28)$ \\
\hline & Culex decens & $4.3(1)$ & $0.0(0)$ & $6.5(4)$ & $0.0(0)$ & $0.0(0)$ & $0.0(0)$ & $0.0(0)$ & $2.0(5)$ \\
\hline & Culex nebulosus & $26.1(6)$ & $26.7(12)$ & $16.4(10)$ & $4.6(4)$ & $0.0(0)$ & $0.0(0)$ & $0.0(0)$ & $12.5(32)$ \\
\hline & Culex perfuscus & $0.0(0)$ & $0.0(0)$ & $0.0(0)$ & $2.3(2)$ & $0.0(0)$ & $0.0(0)$ & $0.0(0)$ & $0.8(2)$ \\
\hline & Culex tritaeniorhynchus & $0.0(0)$ & $0.0(0)$ & $0.0(0)$ & $0.0(0)$ & $3.3(1)$ & $0.0(0)$ & $0.0(0)$ & $0.4(1)$ \\
\hline & Eretmapodites chrysogaster & $4.3(1)$ & $2.2(1)$ & $8.2(5)$ & $2.3(2)$ & $3.3(1)$ & $0.0(0)$ & $0.0(0)$ & $3.9(10)$ \\
\hline \multirow[t]{3}{*}{ Bamboo holes } & Aedes aegypti & na & na & $42.8(3)$ & $55.5(5)$ & $0.0(0)$ & na & na & $40.8(8)$ \\
\hline & Aedes unilineatus & na & na & $0.0(0)$ & $22.2(2)$ & $0.0(0)$ & na & na & $10.0(2)$ \\
\hline & Eretmapodites chrysogaster & na & na & $0.0(0)$ & $0.0(0)$ & $25(1)$ & na & na & $5.0(1)$ \\
\hline \multirow[t]{8}{*}{ Rock holes } & Aedes aegypti & $0.0(0)$ & $1.4(1)$ & $0.0(0)$ & $0.0(0)$ & $0.0(0)$ & $0.0(0)$ & na & $0.3(1)$ \\
\hline & Aedes unilineatus & $1.4(1)$ & $0.0(0)$ & $0.0(0)$ & $0.0(0)$ & $0.0(0)$ & $0.0(0)$ & na & $0.3(1)$ \\
\hline & Aedes vittatus & $2.8(2)$ & $7.1(5)$ & $7.1(5)$ & $1.4(1)$ & $10.3(3)$ & $20(2)$ & na & $5.6(18)$ \\
\hline & Culex decens & $1.4(1)$ & $1.4(1)$ & $1.4(1)$ & $0.0(0)$ & $0.0(0)$ & $40(4)$ & na & $2.2(7)$ \\
\hline & Culex nebulosus & $0.0(0)$ & $1.4(1)$ & $1.4(1)$ & $0.0(0)$ & $0.0(0)$ & $10(1)$ & na & $0.9(3)$ \\
\hline & Culex perfuscus & $1.4(1)$ & $1.4(1)$ & $0.0(0)$ & $0.0(0)$ & $3.4(1)$ & $20(2)$ & na & $1.6(5)$ \\
\hline & Culex tritaeniorhynchus & $0.0(0)$ & $1.4(1)$ & $0.0(0)$ & $0.0(0)$ & $6.9(2)$ & $0.0(0)$ & na & $0.9(3)$ \\
\hline & Eretmapodites chrysogaster & $0.0(0)$ & $0.0(0)$ & $1.4(1)$ & $0.0(0)$ & $3.4(1)$ & $0.0(0)$ & na & $0.6(2)$ \\
\hline Tires & Ae. aegypti & $0.0(0)$ & $0.0(0)$ & $0.0(0)$ & $75(3)$ & $0.0(0)$ & na & $0.0(0)$ & 27.3 (3) \\
\hline
\end{tabular}

Number of microhabitats positive for each data entry is in parentheses.

\section{Species richness diversity and dominance}

The species richness, diversity and dominances in the different land covers and microhabitats are presented in Table 3. The highest number of species and diversity were observed in the forest for the land covers $(\mathrm{p} \leq$ $0.02)$ and the tree holes for the microhabitats $(\mathrm{p} \leq 0.001)$ in this survey. Dominance in the forest was significantly less than in the other land covers $(\mathrm{p} \leq 0.001)$. Among the microhabitats, tree holes had the lowest dominance $(\mathrm{p} \leq 0.001)$. The following analyses take into account only the common species comprising more than $1 \%$ of the total mosquito fauna.

\section{Larval frequencies of occurrence and dynamics of common species}

Larval occurrence in the different land cover classes and microhabitat types varied by species (Table 4). Aedes aegypti, Cx. nebulosus, Cx. perfuscus, Cx. tritaeniorhynchus,
Er. chrysogster and Ae. vittatus were collected from all positive land cover classes, whereas Ae. furcifer and Ae. taylori were found only in forests and savannahs; among the other common species in forests, savannahs and villages (Table 2). Aedes aegypti was the only common species collected in all the 9 microhabitats encountered in this study, whereas $A e$. furcifer and Ae. taylori were present in fresh fruit husks and tree holes (Table 3); the other common species were more evenly distributed.

Aedes furcifer immature stages were significantly more likely to be encountered in savannahs $\left(\chi^{2}=3.96\right.$; $\mathrm{df}=1$; $\mathrm{p}=0.04)$, and Ae. longipalpis and $C x$. cinerus in forests $(\mathrm{p} \leq 0.007)$, whereas some others species (Ae. luteocephalus, Ae. unilineatus, Cx. decens, Er. Chrysogaster and Ae. taylori) were found almost equally in forests and savannahs $(\mathrm{p} \geq 0.06)$. Aedes aegypti and Cx. tritaeniorhychus were equally likely to occur in savannahs and villages $(\mathrm{p} \geq 0.07)$. Aedes vittatus and $C x$. nebulosus, in 
Table 4 Abundance of immature stages of common mosquito species in different microhabitats, in sylvatic arbovirus foci, in Kédougou from June - December 2010

\begin{tabular}{|c|c|c|c|c|c|c|c|c|c|}
\hline Species & $\begin{array}{l}\text { Decaying } \\
\text { fruit husks }\end{array}$ & $\begin{array}{l}\text { Fresh } \\
\text { fruit husks }\end{array}$ & Puddles & $\begin{array}{l}\text { Discarded } \\
\text { containers }\end{array}$ & $\begin{array}{l}\text { Tree } \\
\text { holes }\end{array}$ & $\begin{array}{l}\text { Bamboo } \\
\text { holes }\end{array}$ & $\begin{array}{l}\text { Rock } \\
\text { holes }\end{array}$ & Tires & $\begin{array}{l}\text { Storage } \\
\text { containers }\end{array}$ \\
\hline Aedes aegypti & $1.37^{\mathrm{a}}$ & $0.2^{\mathrm{b}}$ & $0.14^{b}$ & $0.77^{\mathrm{a}, \mathrm{b}}$ & $0.14^{b}$ & $0.88^{\mathrm{a}, \mathrm{b}}$ & $0.02^{c}$ & $0.6^{a, b}$ & $0.02^{c}$ \\
\hline Aedes furcifer & & $0.02^{b}$ & & & $0.13^{\mathrm{a}}$ & & & & \\
\hline Aedes longipalpis & & $0.005^{b}$ & $0.01^{a, b}$ & & $0.14^{\mathrm{a}}$ & & & & \\
\hline Aedes luteocephalus & $0.1^{\mathrm{a}, \mathrm{b}}$ & $0.05^{\mathrm{b}}$ & $0.04^{b}$ & $0.03^{b}$ & $0.35^{\mathrm{a}}$ & & & & \\
\hline Aedes taylori & & $0.01^{\mathrm{b}}$ & & & $0.3^{\mathrm{a}}$ & & & & \\
\hline Aedes unilineatus & $0.23^{b}$ & $0.06^{\mathrm{b}}$ & & $0.006^{c}$ & $0.12^{\mathrm{a}}$ & $0.09^{a, b}$ & $0.002^{c}$ & & \\
\hline Aedes vittatus & & $0.014^{b}$ & $0.44^{\mathrm{a}}$ & $0.05^{\mathrm{b}}$ & $0.02^{\mathrm{b}}$ & & $0.11^{\mathrm{b}}$ & & \\
\hline Culex cinerus & $0.05^{a, b}$ & $0.03^{b}$ & $0.06^{\mathrm{a}, \mathrm{b}}$ & $0.03^{b}$ & $0.16^{\mathrm{a}}$ & & & & \\
\hline Culex decens & & 0.005 & 0.07 & 0.02 & 0.02 & & 0.04 & & \\
\hline Culex nebulosus & $0.1^{\mathrm{a}}$ & $0.1^{\mathrm{a}}$ & $0.15^{\mathrm{a}}$ & $0.16^{\mathrm{a}}$ & $0.19^{\mathrm{a}}$ & & $0.009^{b}$ & & \\
\hline Culex perfuscus & & 0.005 & 0.15 & 0.04 & 0.05 & & 0.03 & & \\
\hline Culex tritaeniorhynchus & $0.07^{\mathrm{a}}$ & $0.009^{b}$ & $0.1^{\mathrm{a}}$ & $0.2^{\mathrm{a}}$ & $0.003^{b}$ & & $0.006^{b}$ & & \\
\hline Eretmapodites chrysogaster & $2.1^{\mathrm{a}}$ & $1.5^{\mathrm{a}}$ & & $0.04^{\mathrm{b}}$ & $0.03^{b}$ & $0.06^{\mathrm{b}}$ & $0.006^{c}$ & & \\
\hline
\end{tabular}

Letters indicate the results of paired Mann-Whitney test when the Kruskal-Wallis test was found statistically significant or when only two habitats were being compared. Groups that do not share a letter are significantly different $(P<0.05)$.

contrast, occurred at statistically comparable frequencies in all four land cover classes $(p \geq 0.1)$.

Not all of the species analyzed had comparable frequencies among microhabitats. Ae. furcifer, Ae. longipalpis and Ae. taylori were most frequent in tree holes ( $\mathrm{p} \leq 0.001)$, Ae. vittatus and $C x$. perfuscus in puddles ( $\mathrm{p} \leq 0.0002$ ). The other species were more evenly distributed and were detected in higher and statistically comparable frequencies in different combinations of microhabitats. Indeed, $A e$. aegypti was detected in higher and comparable frequencies in decaying fruit husks, bamboo holes, tires and discarded containers, Ae. luteocephalus in tree holes and decaying fruit husks, Ae. unilineatus in tree holes, bamboo holes and decaying fruit husks, Er. Chrysogaster in decaying fruit husks and fresh fruit husks, $C x$. cinerus in decaying fruit husks, tree holes and puddles, $C x$. nebulosus in decaying fruit husks, fresh fruit husks, puddles, discarded containers and tree holes, $C x$. tritaeniorhychus in decaying fruit husks, puddles and discarded containers and finally $C x$. decens in puddles, discarded containers, tree holes and rock holes ( $\mathrm{p} \geq 0.09$ ).

Immature stages of twelve of the thirteen most common species appeared for the first time in June in the forest land cover for Ae. furcifer, Ae. taylori, Ae. luteocephalus, Ae. unilineatus, $C x$. decens and Er. chrysogaster, in the forest and the savannah for Ae. vittatus and in forest and village for Ae. aegypti, Ae. longipalpis, Cx. cinerus, Cx. nebulosus and Cx. perfuscus (Table 4). Culex tritaeniorhynchus appeared for the first time only in July in the village, barren and savannah land covers. These species were collected for the last time in December for Ae. luteocephalus, Ae. unilineatus and Cx. nebulosus, in
November for Ae. aegypti, Ae. vittatus and Cx. perfuscus and in October for the other species.

\section{Larval abundance by species}

The abundance of each species was compared among land cover or infested microhabitat types where they occurred. The analysis revealed statistically significant variations in larval abundances in the different land cover classes for all species except Ae. taylori $(U=24844, \mathrm{Z}=-0.3, \mathrm{p}=0.8)$ and Ae. vittatus $(H=5.9, \mathrm{p}=0.1)$ (Figure 5$)$. Aedes aegypti larvae were most abundant in villages and savannahs, Ae. luteocephalus, Ae. unilineatus and Er. chrysogaster in savannahs and forests, $C x$. nebulosus in savannahs, forests and villages, Ae. longipalpis, $C x$. cinerus and $C x$. decens in forests ( $\mathrm{p} \geq 0.08)$ and Ae. furcifer in savannahs ( $<<0.05)$.

Among microhabitats, there were statistically significant variations in larval abundances for all the species except Cx. decens $(H=2.2, \mathrm{p}=0.7)$ and $C x$. perfuscus $(H=9.4$, $\mathrm{p}=0.05)$ (Table 5). Aedes furcifer $(U=17923.5, \mathrm{Z}=-3.3$, $\mathrm{p}=0.001)$, Ae. taylori $(U=18952.5, \mathrm{Z}=-4.6, \mathrm{p}<0.0001)$ and $C x$. nebulosus $(\mathrm{p}<0.05)$ were most abundant in tree holes, Ae. vittatus in puddles and tree holes, Ae. luteocephalus in tree holes and decaying fruit husks, Ae. longipalpis in tree holes and puddles, Ae. unilineatus in decaying fruit husks, tree holes and bamboo holes, Er. chrysogaster in decaying fruit husks and fresh fruit husks, $C x$. cinerus in tree holes, decaying fruit husks and puddles, $C x$. tritaeniorhynchus in discarded containers, decaying fruit husks and puddles and finally Ae. aegypti in decaying fruit husks, discarded containers, bamboo holes and tires $(\mathrm{p} \geq 0.07)$. 


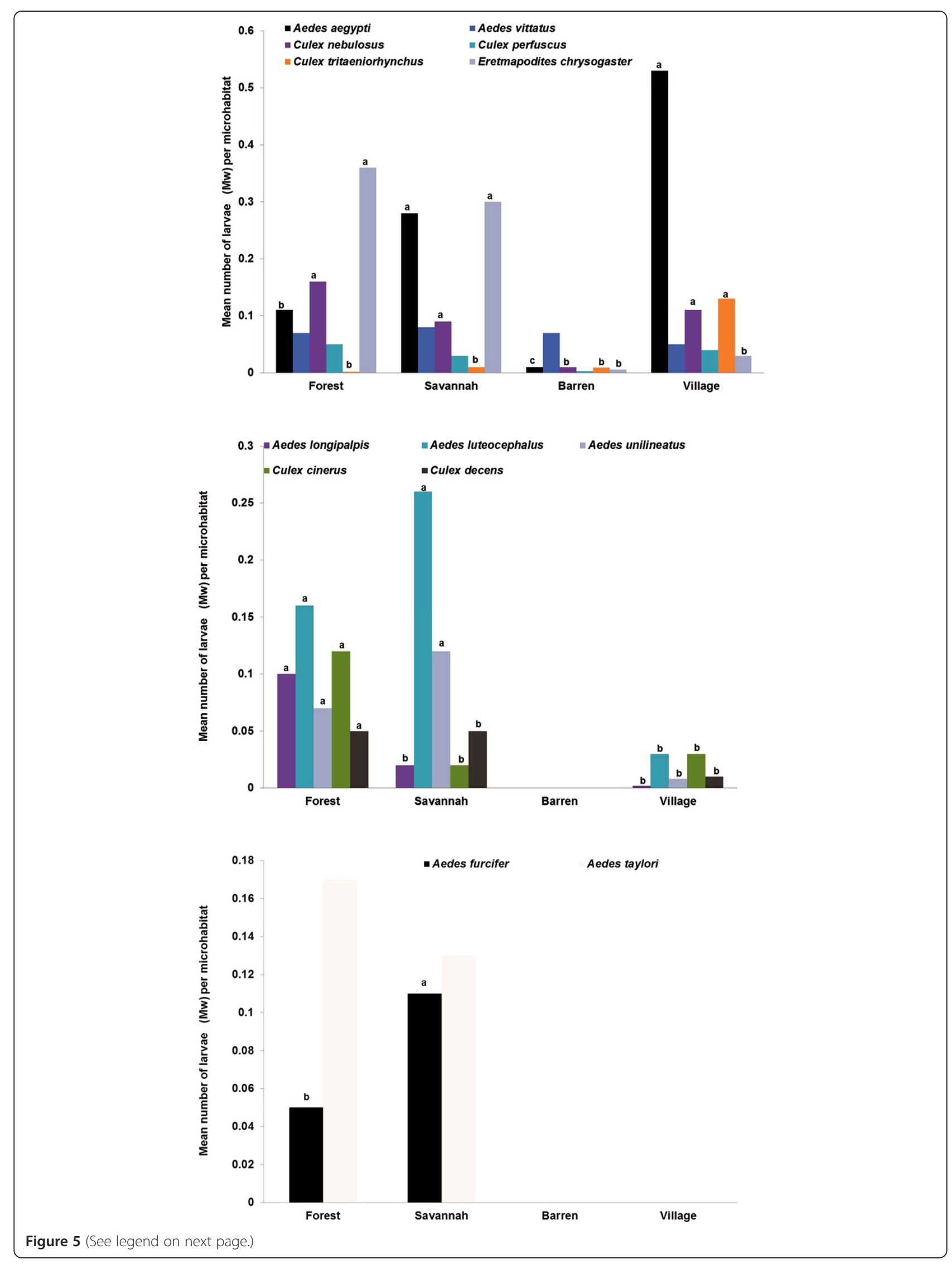


(See figure on previous page.)

Figure 5 Abundance of immature stages (larvae/ wet container) of common mosquito species in different land cover classes, foci of sylvatic arbovirus, in Kédougou from June - December 2010. Letters indicate the results of a paired Mann-Whitney test when the Kruskal-Wallis test was found statistically significant or when only two habitats were being compared. Groups that do not share a letter are significantly different $(P<0.05)$.

\section{Interspecific association of the common species}

The 66 pairings between the 12 most common species (Table 6) revealed 15 significant associations (10 positive and 5 negative associations). The highest positive and significant associations were between Ae. luteocephalus and Ae. furcifer, Ae. taylori and Ae. furcifer, and Ae. luteocephalus and Ae. longipalpis. All the five significant negative associations involved Er. Chrysogaster with Ae. luteocephalus, Ae. taylori, Ae. longipalpis, Cx. cinerus and Cx. decens.

\section{Discussion}

Our larval collections yielded 15 more mosquito species than the only previous larval survey in the Kédougou region [13]. This higher number of species is likely due to the greater number of forested sites and more diverse land cover classes that we investigated. Indeed, the previous study [13] focused only on a single gallery forest, while we sampled forests, savannahs, barren areas and villages.

Only about one-third of the available water-filled microhabitats were occupied by immature mosquitoes, suggesting the possibility that gravid females choose their oviposition sites carefully. Similar findings have been reported by previous investigations [24-26]. Among others, the location of the microhabitat, its color, the chemical composition of the water, the quality and availability of food may be important factors determining mosquito frequencies of occurrence in water-filled microhabitats [27]. Recently, Wong et al. [28] also documented that Ae. aegypti exhibits strong conspecific attraction during oviposition site selection.

Larvae were detected between June and OctoberDecember, depending on the species. This pattern indicated clearly that rainfall is a key factor in larval ecology in Kédougou. Indeed, all the natural and artificial microhabitats used as larval habitats were filled by rainfall.

It was noteworthy that immature Ae. furcifer and Ae. taylori, two of the main YF, DENV-2 and CHIKV vectors, were collected in a very restricted range of habitats in contrast to Ae aegypti and Ae. vittatus, which were found in a wide range of habitats. Aedes luteocephalus had a slightly different pattern of larval distribution. It was collected mainly in forests and savannahs within tree holes and fruit husks but was also collected in villages, albeit at a lower frequency.

The positive associations between Ae. furcifer, Ae. luteocephalus and Ae. taylori suggest that their gravid females follow the same ovipositional stimuli and illustrate their common larval preference for tree holes that probably have attractive physical and chemical elements for these species. Negative associations between Er. Chrysogaster and some species may be due, at least in part, to different ovipositional stimuli or to kairomonal repellents emitted by this species against the others. The same chemical product may be a stimulus to its conspecific.

Like the observation of Bang et al. [29] in Nigeria, but contrary to what was observed in East Africa [30], we detected a high diversity of mosquito larvae in domestic environments, although it was lower than the diversity in the forest. The higher species richness in tree holes may be due to their higher stability and trophic richness compared to the other microhabitats. Indeed, tree holes retained water for longer periods of time than the other microhabitats, which made them ideal larval sites for more species. Immatures in the tree holes may also be better protected against flushing during heavy rains.

The high occurrence of larval Ae. aegypti formosus in villages in the Kédougou region was not expected, because this subspecies is reported to undergo larval development in the forest, specifically in tree holes, rock holes and fruit husks [31]. However, its larvae have been found indoors in villages in Nigeria [32] and Gabon [33]. Discarded containers were among the main habitats for immature Ae. aegypti in villages in our study, suggesting a strong impact of human activities on the distribution of this species. The high container index of Ae. aegypti may suggest that the area is at high risk of YFV and other sylvatic arbovirus epidemics. However, human landing data $[2,12,14]$ indicate that this species is minimally attracted to humans in the area. Virus isolations and vector competence studies also indicate that this species is rarely associated with arbovirus infection and has a low susceptibility to DENV-2 [2,14,34,35]. Thus, Ae. aegypti larval indices should be interpreted with caution in epidemiological risk evaluation for some rural areas of Africa because peridomestic larval habitats may be occupied by a highly zoophilic population of Ae. aegypti formosus. Despite a high degree of water storage, making many containers available as potential larval habitats, only one clay pot was found occupied by $A e$. aegypti formosus during our study. This may indicate the sylvatic nature of this species and/or that adaption to peridomestic environments is ongoing. Our data thus suggest that removal of discarded containers in villages will allow efficient control of Ae. aegypti, but will likely have little impact on sylvatic arbovirus transmission 
Table 5 Richness and dominance of mosquito species in different land covers and microhabitats, within foci of sylvatic arbovirus, in Kédougou from June - December 2010

\begin{tabular}{llll}
\hline Macrohabitat & Richness & Dominance & $\begin{array}{l}\text { Shannon } \\
\text { diversity }\end{array}$ \\
\hline Forest & $31^{\mathrm{a}}$ & $0.11^{\mathrm{c}}$ & $2.52^{\mathrm{a}}$ \\
Savannah & $21^{\mathrm{b}}$ & $0.12^{\mathrm{b}}$ & $2.36^{\mathrm{b}}$ \\
Barren & $11^{\mathrm{b}}$ & $0.37^{\mathrm{a}}$ & $1.47^{\mathrm{c}}$ \\
Village & $19^{\mathrm{b}}$ & $0.34^{\mathrm{a}}$ & $1.52^{\mathrm{c}}$ \\
Microhabitat & & & \\
Decaying fruit husks & $9^{\mathrm{c}}$ & $0.39^{\mathrm{b}}$ & $1.21^{\mathrm{d}}$ \\
Fresh fruit husks & $19^{\mathrm{b}}$ & $0.4^{\mathrm{b}}$ & $1.25^{\mathrm{d}}$ \\
Puddles & $11^{\mathrm{c}}$ & $0.31^{\mathrm{c}}$ & $1.63^{\mathrm{b}}$ \\
Discarded containers & $18^{\mathrm{b}}$ & $0.33^{\mathrm{c}}$ & $1.5^{\mathrm{c}}$ \\
Tree holes & $28^{\mathrm{a}}$ & $0.12^{\mathrm{d}}$ & $2.42^{\mathrm{a}}$ \\
Bamboo holes & $3^{\mathrm{d}}$ & $0.82^{\mathrm{a}}$ & $0.38^{\mathrm{e}}$ \\
Rock holes & $15^{\mathrm{b}}$ & $0.29^{\mathrm{c}}$ & $1.67^{\mathrm{b}}$ \\
Tires & $2^{\mathrm{d}}$ & $0.92^{\mathrm{a}}$ & $0.17^{\mathrm{a}}$ \\
Storage containers & $1^{\mathrm{d}}$ & $1^{\mathrm{a}}$ & $0^{\mathrm{f}}$ \\
\hline
\end{tabular}

Groups that do not share a letter are significantly different (Bootstrap; $\mathrm{P}<0.05)$.

because Ae. furcifer, the main YFV, CHIKV and DENV-2 vector in this region, primarily lays eggs in the forest and savannah.

Our data indicated that the proportion of Ae. taylori was much higher than that of Ae. furcifer in the immature fauna, while the opposite was always observed in human landing fauna $[2,14,36]$. This discordance suggests that a part of the population of Ae. taylori is not anthropophilic, or that the population of immature Ae. furcifer was incompletely sampled due to our failure to identify its preferred larval sites. Thus, the Ae, taylori population may be more important than indicated by human landing catch data. Moreover, if we consider that non-anthropophilic mosquitos are generally also non-primatophilic [37], a portion of the Ae. taylori population may feed in as yet unknown hosts in the forest. Our possible failure to fully identify the preferred larval sites of Ae. furcifer may be due to the fact that we sampled only visible and readily accessible tree holes (located at less than $2 \mathrm{~m}$ above the ground) while some tree holes were located more than $10 \mathrm{~m}$ high. These elevated tree holes may be preferred larval habitats for $A e$. taylori; height-dependent oviposition behavior has been already observed in African forests [15,38,39] and in Indiana [40]. This hypothesis requires further investigation. Although Ae. furcifer was the main sylvatic arbovirus (YF, DENV-2 and CHIKV) vector collected by human landing collections and was the only species found infected in villages in Africa [2,14,41], its larvae were not found within these villages. Therefore, the adult females of this species probably invade villages each evening from savannahs and/or forests, where we found its larvae in tree holes and fruit husks. A more detailed understanding of the movement of Ae. furcifer between larval habitats and human habitations will yield a better understanding of how people are exposed to sylvatic YFV, DENV-2 and CHIKV.

We found Ae. vittatus larvae in all land cover classes and in 5 of the 9 microhabitats sampled. Its most common larval habitats were puddles followed by rock holes in this study. Although it has been already found in

Table 6 Coefficients of interspecific association $\left(C_{7}\right)$ for the most common mosquito species in foci of sylvatic arbovirus in Kédougou from June - December 2010

\begin{tabular}{|c|c|c|c|c|c|c|c|c|c|c|c|c|}
\hline & aegypti & furcifer & longi & luteo & taylori & unilin & vittatus & cinerus & decens & nebulo & perfus & chryso \\
\hline furcifer & 0.06 & & & & & & & & & & & \\
\hline longi & 0.05 & 0.1 & & & & & & & & & & \\
\hline luteo & 0.2 & $0.5^{* * *}$ & 0.1 & & & & & & & & & \\
\hline taylori & 0.07 & $0.4^{* * *}$ & $0.4^{* * *}$ & $0.4^{* * *}$ & & & & & & & & \\
\hline unilin & 0.08 & -0.1 & -0.4 & $0.3^{* *}$ & 0.04 & & & & & & & \\
\hline vittatus & -0.3 & 0.01 & -0.2 & -0.7 & -0.4 & -0.06 & & & & & & \\
\hline cinerus & 0.1 & -0.04 & $0.2^{*}$ & 0.04 & $0.3^{* * *}$ & -0.4 & 0.01 & & & & & \\
\hline decens & 0.1 & -0.1 & -1 & -01 & -0.5 & -0.3 & 0.09 & 0.02 & & & & \\
\hline nebulo & $0.3^{*}$ & 0.03 & 0.06 & 0.08 & 0.1 & 0.1 & -0.7 & $0.4^{* * *}$ & -0.03 & & & \\
\hline perfus & 0.4 & -1 & -1 & -1 & -0.5 & -0.2 & 0.1 & -1 & $0.5^{* * *}$ & -1 & & \\
\hline chryso & 0.3 & -0.3 & $-0.7^{* *}$ & $-0.6^{* *}$ & $-0.5^{* *}$ & -0.1 & -0.4 & $-0.6^{* *}$ & $-1^{*}$ & -0.3 & -0.7 & \\
\hline tritaenio & -0.2 & -1 & -1 & -1 & -1 & -1 & -1 & -1 & -1 & -1 & -1 & 1 \\
\hline
\end{tabular}

Aegypti $=$ Ae. aegypti, furcifer $=$ Ae. furcifer, longi $=$ Ae. longipalpis, luteo $=$ Ae. luteocephalus, taylori $=A$ e. taylori, unilin $=$ Ae. unilineatus, vittatus $=A e$. vittatus, cinerus $=C x$. cinerus, decens $=C x$. decens, nebulo $=C x$. nebulosus, perfus $=C x$. perfuscus and tritaenio $=C x$. tritaeniorynchus. Levels of significance for $x^{2}:{ }^{*} p<0.05,{ }^{* *} p<0.01$, *** $\mathrm{p}<0.001$. 
puddles in Midwestern Nigeria [42], the larvae of this species are generally known to occupy rock holes in Africa [43,44].

Our data agree with previous studies showing that $A e$. luteocephalus larvae are generally found in natural tree holes and in low frequencies in various water containers in villages $[24,30,39,45]$. This mosquito has been collected year-round in Nigeria [39] and its larvae have been collected 4 months after the last seasonal rainfall in the Kédougou region [31]. Thus, Ae. luteocephalus may be considered particularly tolerant of dry conditions [39] but may stop larval development in response to a lack of wet tree holes. Our data also suggest that the Aedes of the africanus group found by Raymond et al. [13] were probably Ae. luteocephalus.

The preference of this species to oviposit in tree holes is in agreement with the findings of Dunn [46] and Anosike et al. [47] in Nigeria. However, another investigator also found this species in water containers within villages in the same country [48].

Other mosquitoes like Er. chrysogaster and Ae. longipalpis were also highly represented in the immature fauna we collected, while they were scarce or absent from all previous 25-hour [12] and crepuscular [2,14] human landing collections in the area. CHIKV or DENV-2 strains have not been isolated from these species under natural conditions, but they may be considered as potential vectors. Indeed, Er. chrysogaster has been shown experimentally to have a higher vector potential for CHIKV than Ae aegypti [49,50]. Ae. longipalpis belongs to the same subgenus as Ae. niveus and Ae. ingrami, which are sylvatic DENV vectors in Malaysia [51] and potential CHIKV vectors in the Ivory coast [52], respectively. The presence of large populations of non-anthropophilic Ae. taylori, Er. chrysogaster and Ae. longipalpis suggest the existence of an as-yet undescribed secondary enzootic cycle of DENV-2 and CHIKV.

\section{Conclusions}

Our study provides valuable information on the larval ecology of sylvatic arbovirus vectors in southeastern Senegal. We have shown that Ae. furcifer and Ae. taylori larvae occur mainly in tree holes in forest and savannah land covers, unlike immature Ae aegypti and Ae. vittatus, which were found in a wider range of microhabitats and land cover classes. Ae. luteocephalus was collected mainly in forest and savannah land covers within tree holes and fruit husks but was also collected in a lower frequency in various containers in villages. Larvae of zoophilic $A e$. aegypti formosus were frequently found in discarded containers in villages. We also detected a high proportion of non-anthropophilic potential vectors in the larval mosquito fauna suggesting the existence of still obscure YFV, DENV-2 and CHIKV cycles in southeastern Senegal.
Removal of discarded containers will be efficient for controlling Ae. aegypti in villages but will have little or no impact in Ae. furcifer, the principal DENV and CHIKV vector to humans.

\section{Competing interests}

The authors declare that they have no competing interests.

\section{Authors' contributions}

$M D, S C W, A A S$ and $K A H$ conceived the study. DD designed the protocol. MB carried the remote sensing and geospatial analysis. DD and CTD carried out the field work. DD and MD analyzed the data and drafted the manuscript. $\mathrm{DD}, \mathrm{CTD}, \mathrm{MB}, \mathrm{KAH}, \mathrm{SCW}, \mathrm{ID}, \mathrm{YB}$ and MD critically revised the manuscript. All authors read and approved the final manuscript.

\section{Acknowledgements}

This study was supported by grants from the National Center for Research Resources (5P20RR016480-12), the National Institute for Allergy and Infectious Diseases (R01-Al069145) and the National Institute of General Medical Sciences (8 P20 GM103451-12) from the National Institutes of Health. The authors would like to thank Saliou Ba, Omar Ba, Lamine Traoré and Bidiel Fall for their technical assistance in the field and all the population of Kédougou for their collaboration.

\section{Author details}

'Unité d'entomologie médicale, Institut Pasteur de Dakar, Dakar, Sénégal. 2Department of Biology, New Mexico State University, Las Cruces, New Mexico, United States of America. ${ }^{3}$ Unité des arbovirus et virus des fièvres hémorragiques, Institut Pasteur de Dakar, Dakar, Sénégal. ${ }^{4}$ Department of Geography, New Mexico State University, Las Cruces, New Mexico, United States of America. ${ }^{5}$ Institute for Human Infections and Immunity, Center for Biodefense and Emerging Infectious Diseases, and Department of Pathology, University of Texas Medical Branch, Galveston, Texas, United States of America.

Received: 13 August 2012 Accepted: 3 December 2012

Published: 7 December 2012

\section{References}

1. Diallo M, Thonnon J, Traore-Lamizana M, Fontenille D: Vector of the Chikungunya virus in Senegal: current date and transmission cycles. AmJTrop Med Hyg 1999, 60:281-286.

2. Diallo M, Ba Y, Sall AA, Diop OM, Ndione JA, Mondo M, Girault L, Mathiot C: Amplification of the sylvatic cycle of dengue virus type 2, Senegal, 1999-2000: entomologic findings and epidemiologic considerations. Emerg Infect Dis 2003, 9(3):362-367.

3. Cornet M, Robin Y, Château R, Heme G, Adam C, Valade M: Isolement d'arbovirus au Sénégal oriental à partir de moustiques (1972-1977) et note sur l'épidémiologie des virus transmis par les Aedes, en particulier du virus amaril. Cah ORSTOM Ser Ent Med Parasitol 1979, 17:149-163.

4. Robin $Y$, Cornet M, Hème G, Legonidec $G$ : Isolement du virus de la dengue au Sénégal. Ann. Virol(Inst. Pasteur) 1980, 131E:149-154.

5. Cornet M, Saluzzo JF, Hervy JP, Digoutte JP, Germain M, Chauvancy MF, Eyraud M, Ferrara L, Heme G, Legros F: Dengue 2 au Sénégal oriental: une pousse épizootique en milieu selvatique; isolements du virus à partir de moustiques et d'un singe et considérations épidémiologiques. Cah ORSTOM Ser Ent Med Parasitol 1984, 22:313-323.

6. Cornet M: Dengue in Africa, Epidemiology of dengue and dengue haemorrhagic fever. Monograph on dengue/dengue hemorrhagic fever. World Health Organ 1993, 22:39-47.

7. Saluzzo JF, Cornet M, Castagnet P, Rey C, Digoutte JP: Isolation of dengue 2 and dengue 4 viruses from patients in Senegal. Trans R Soc Trop Med Hyg 1986, 80:5.

8. Zeller HG, Traore-Lamizana M, Monlun E, Hervy JR, Mondo M, Digoutte JR: Dengue-2 virus isolation from humans during an epizootic in southeastern Senegal in November, 1990. Res Virol 1992, 143:101-102.

9. Taufflieb R, Cornet M, Le Gonidec G, Robin Y: Un foyer selvatique de fièvre jaune au Sénégal oriental. Cah ORSTOM Ser Ent Med Parasitol 1973, 3:211-220. 
10. Robin Y, Taufflieb R, Cornet M: Passé, présent et devenir possible de l'épidémiologie de la fièvre jaune et de sa surveillance en Afrique de I'Ouest francophone. Cah ORSTOM Ser Ent Med Parasitol 1972, 2:95-97.

11. Traore-Lamizana M, Fontenille D, Zeller HG, Mondo M, Diallo M, Adam F, Eyraud M, Maiga A, Digoutte JP: Surveillance for yellow fever virus in eastern Senegal during 1993. J Med Entomol 1996, 33(5):760-765.

12. Cornet M, Chateau R, Valade M, Dieng PY, Raymond H, Lorand A: Données bio-écologiques sur les vecteurs potentiels de virus amaril. Cah ORSTOM Ser Ent Med Parasitol 1978, 16:315-341.

13. Raymond $\mathrm{H}$, Cornet M, DIENG PY: Etudes sur les vecteurs sylvatiques du virus amaril. Inventaire provisoire des habitats larvaires d'une forêtgalerie dans le foyer endémique du Sénégal Oriental. Cah ORSTOM Ser Ent Med Parasitol 1976, 14:301-306.

14. Diallo D, Sall AA, Buenemann M, Chen R, Faye O, Diagne CT, Ba Y, Dia I, Watts D, Weaver SC, Hanley KA, Diallo M: Landscape ecology of sylvatic chikungunya virus and mosquito vectors in southeastern senegal. PLoS Negl Trop Dis 2012, 6:e1649.

15. Lounibos L: Habitat segregation among African treehole mosquitoes. Ecol Entomol 1981, 6:129-154.

16. Edwards FW: Mosquitoes of the Ethiopian Region. III. Culicine Adults and Pupae. London: British Museum of Natural History; 1941

17. Ferrara L, Germain M, Hervy JP: Aedes (Diceromyia) furcifer (Edwards, 1913) et Aedes (Diceromyia) taylori (Edwards, 1936): le point sur la différentiation des adultes. Cah ORSTOM Ser Ent Med Parasitol 1984, 22:95-98.

18. Huang YM: Aedes (Stegomyia) bromeliae (Diptera: Culicidae), the yellow fever virus vector in East Africa. J Med Entomol 1986, 23:196-200.

19. Jupp PG: Mosquitoes of southern Africa: Culicinae and Toxorhynchitinae Hartebeespoort. South Africa: Ekogilde cc Publishers; 1997

20. Diagne N, Fontenille D, Konate L, Lamizana MT, Molez JF, Trape JF, Faye O: Les Anophèles du Sénégal: liste comment \& et illustrée. Bull Soc Pathol Exot 1994, 87:1-9.

21. Williams CB: The use of logarithms in the interpretation of certain entomological problems. Ann Appl Biol 1937, 24:404-414.

22. Cole LC: Measurement of interspecific association. Ecology 1949, 30:411-424.

23. Pielou EC: Mathematical Ecology. New York: John Wiley \& Sons; 1977

24. Rickenbach A, Button JP: Enquête sur les vecteurs potentiels domestiques de fièvre jaune au Cameroun. Cah ORSTOM Ser Ent Med Parasitol 1977, 15:93-103.

25. Adeleke MA, Mafiana CF, Idowu AB, Adekunle MF, Sam-Wobo SO: Mosquito larval habitats and public health implications in Abeokuta, Ogun State, Nigeria. Tanzania J Health Res 2008, 10(2):103-108.

26. Chambers DM, Young LF, Hill HS Jr: Backyard mosquito larval habitat availability and use as influenced by census tract determined resident income levels. J A mosq Contr Assoc 1986, 2:539-544.

27. Lounibos LP: Mosquito breeding and oviposition sitimulant in fruit husk Ecol Entomol 1978, 3:299-304

28. Wong J, Stoddard ST, Astete H, Morrison AC, Scott TW: Oviposition site selection by the dengue vector Aedes aegypti and its implications for dengue control. PLoS Negl Trop Dis 2011, 5(4):e1015.

29. Bang $\mathrm{YH}$, Bown DN, Onwubiko AO: Prevalence of larvae of potential yellow fever vectors in domestic containers in Southern Nigeria. Bull World Health Organ 1981, 59:107-114.

30. Mwangangi JM, Muturi EJ, Mbogo CM: Seasonal mosquito larval abundance and composition in Kibwezi, lower eastern Kenya. J Vector Ecol 2009, 46(1):65-71.

31. Cornet M, Raymond H, Dieng PY: Etudes sur les vecteurs sylvatiques du virus amaril. Essai de mise en évidence de la survie de femelles en saison sèche dans un foyer endémique au Sénégal Oriental. Cah ORSTOM Ser Ent Med Parasitol 1975, 13:215-222.

32. Nasidi A, Monath TP, Decock K, Tomori O, Cordellier R, Olaleye OD, Harry TO, Adeniyi JA, Sorungbe AO, Ajose-Coker AO: Urban Yellow-Fever Epidemic in Western Nigeria, 1987. Trans R Soc Trop Med Hyg 1989, 83:401-406.

33. Vazeille-Falcoz M, Failloux AB, Mousson L, Elissa N, Rodhain F: Ora receptivity of Aedes aegypti formosus from Franceville (Gabon, Central Africa) for dengue type 2 virus. Bull Soc Pathol Exot 1999, 92:341-342.

34. Diallo M, Sall AA, Moncayo AC, Ba Y, Fernandez Z, Ortiz D, Coffey LL, Mathiot C, Tesh RB, Weaver SC: Potential role of sylvatic and domestic African mosquito species in dengue emergence. AmJTrop Med Hyg 2005, 73(2):445-449.
35. Diallo M, Ba Y, Faye O, Soumare ML, Dia I, Sall AA: Vector competence of Aedes aegypti populations from Senegal for sylvatic and epidemic dengue 2 virus isolated in West Africa. Trans R Soc Trop Med Hyg 2008, 102(5):493-498.

36. Traore-Lamizana M, Zeller H, Monlun E, Mondo M, Hervy JP, Adam F, Digoutte JP: Dengue 2 outbreak in southeastern Senegal during 1990: virus isolations from mosquitoes (Diptera: Culicidae). J Med Entomol 1994, 31:623-627.

37. Mutebi JP, Barrett AD: The epidemiology of yellow fever in Africa. Microbes Infect 2002, 4(14):1459-1468.

38. Garnham PCC, Highton RB, Harper JO: The mosquitoes of the Kaimosi Forest, Kenya Colony, with special reference to yellow fever. Bull Entomol Res 1946, 36:473-496.

39. Service MW: The ecology of the tree-hole breeding mosquitoes in the northern Guinea Savannah of Nigeria. J Appl Ecol 1965, 2:1-16.

40. Copeland RS, Craig GB: Habitat segregation among treehole mosquitoes (Diptera: Culicidae) in the Great Lakes region of the United States. Ann Entomol Soc Am 1990, 83:1063-1073.

41. Mondet B: Importance d'Aedes (Diceromyia) furcifer Edwards, 1913 (Diptera: Culicidae) parmi les vecteurs potentiels d'arboviroses, dans l'épidémiologie de la fièvre jaune en savane sub-soudanienne de Côte d'Ivoire. Ann Soc Entomol Fr 1997, 33:47-54.

42. Okogun GRA, Anosike JC, Okere AN, Nwoke BEB: Ecology of mosquitoes of midwestern Nigeria. J Vector Borne Dis 2005, 42:1-5.

43. Adebote DA, Oniye SJ, Muhammed YA: Studies on mosquitoes breeding in rock pools on inselbergs around Zaria Northern Nigeria. J Vector Borne Dis 2008, 45:21-28.

44. Service MW: Studies on the biology and taxonomy of Aedes (Steomyia) vittatus (Bigot) (Diptera: Culicidae) in northern Nigeria. Trans R Ent Soc London 1970, 122:101-143.

45. Lee VH, Monath RP, Tomori O, Fagbani A, Wilson DC: Arbovirus studies in Nupeko Forest; a possible natural focus of yellow fever in Nigeria II: Entomological investigations and viruses isolated. Trans Roy Soc Trop Med Hyg 1974, 68:39-43.

46. Dunn LH: Tree-holes and mosquito breeding in West Africa. Bull Entomol Res 1927, 18:139-144.

47. Anosike JC, Nwoke BEB, Okere AN, Oku EE, Asor JE, Emmy-Egbe 1O, Adimike DA: Epidemiology of tree-hole breeding mosquitoes in the tropical rainforest of Imo State, south-east Nigeria. Ann Agric Environ Med 2007, 14:31-38.

48. Bang $\mathrm{YH}$, Bown DN, Arata AA: Ecological studies of Aedes africanus (Diptera: Culicidae) and associated species in Southern Nigeria. J Med Entomol 1980, 17:411-416.

49. Gilotra SK, Shah KV: Laboratory studies on transmission of Chikungunya virus by mosquitoes. Am J Epidemiol 1967, 86:379-385

50. Mangiafico JA: Chikungunya virus infection and transmission in five species of mosquito. AmJTrop Med Hyg 1971, 20:642-645.

51. Rudnick A, Lim TW: Dengue fever studies in Malaysia. Inst Med Res Mal Bull 1986, 23:151-152

52. The African Arbovirus Database: http://www.pasteur.fr/recherche/banques/ CRORA/hotes/hu050021.htm.

doi:10.1186/1756-3305-5-286

Cite this article as: Diallo et al.: Larval ecology of mosquitoes in sylvatic arbovirus foci in southeastern Senegal. Parasites \& Vectors 2012 5:286.

\section{Submit your next manuscript to BioMed Central and take full advantage of:}

- Convenient online submission

- Thorough peer review

- No space constraints or color figure charges

- Immediate publication on acceptance

- Inclusion in PubMed, CAS, Scopus and Google Scholar

- Research which is freely available for redistribution 